PALABRAS CLAVE

Pensiones

Jubilación

Edad

Calidad de la vida

Promedio de vida

Riesgo

Datos estadísticos

Análisis comparativo

Chile
Jaime Ruiz-Tagle

Profesor Adjunto, Departamento de

Economía y Centro de Microdatos

Universidad de Chile

œ jaimert@econ.uchile.cl

Pablo Tapia

Profesor Adjunto de Análisis Estadístico,

Departamento de Administración

Universidad de Chile

œ pablo.tapia.grinen@gmail.com
REVISTA CEPAL 105 DICIEMBRE 2011

\section{Chile: Pensión anticipada, impaciencia y aversión al riesgo}

\author{
Jaime Ruiz-Tagle y Pablo Tapia
}

A

proximadamente un tercio de los chilenos se pensiona de manera anticipada. Al acercarse a la edad de jubilación, los individuos están más conscientes del estado de salud propio de esa edad, lo que reduce la incertidumbre sobre la calidad de vida futura y puede provocar una mayor impaciencia relativa. En este artículo se analiza teóricamente cómo la esperanza de vida futura afecta a la impaciencia relativa y logra inducir una mayor probabilidad de pensión anticipada. Los resultados empíricos para Chile muestran que a mayor esperanza de vida futura, menor es la probabilidad de pensión anticipada. Además, se estudia teóricamente cómo la aversión al riesgo exacerba la impaciencia relativa, debido a la incertidumbre de poder disfrutar de una buena calidad vida en los años futuros. Los resultados empíricos muestran una correlación positiva entre aversión al riesgo y pensión anticipada a través del mecanismo de la esperanza de vida. 


\section{I}

\section{Introducción}

Mientras que con las políticas de pensiones se procura retardar la edad de jubilación, la pensión anticipada es de uso bastante frecuente en Chile. En efecto, del total de personas que decidieron pensionarse en el año 2006, el $35 \%$ lo hicieron en forma anticipada ${ }^{1}$. Según las administradoras de fondos de pensiones (AFP), la edad promedio de los pensionados por vejez anticipada es de 55 años. Desde el punto de vista de las políticas públicas resulta interesante analizar los motivos que condujeron a una persona a tomar tal decisión. En este artículo se presenta una nueva perspectiva en la que se expone cómo la impaciencia y la aversión al riesgo juegan un papel importante en la decisión de adelantar la pensión.

En el caso de pensionarse de manera anticipada, el individuo obtendrá menos recursos económicos que si lo hiciera a la edad legal, con lo que además tendrá que financiar más años de pensión en promedio (Nalebuff y Zeckhauser, 1985). El hecho de que el número de pensionados aumenta cada año y que el $35 \%$ de ellos se pensionan de forma anticipada, hace relevante comprender cómo esta aversión al riesgo influye en la decisión de pensionarse antes de la edad legal ${ }^{2}$.

En Chile, para poder pensionarse anticipadamente ${ }^{3}$ se debe pertenecer al nuevo sistema por un período no menor de cinco años y presentar una pensión que sea igual o superior al $62 \%$ de las rentas imponibles y declaradas en los últimos 10 años $^{4}$. Sin embargo, medidas como esta posiblemente no cambien la condición de impaciencia de las personas.

A la edad en que enfrenta la decisión de pensionarse anticipadamente, o a la edad legal, un individuo está más consciente de la pérdida de capacidad cognitiva y

$\square$ Los autores agradecen los valiosos comentarios de José Luis Ruiz, y a los participantes en el Encuentro de la Sociedad de Economía de Chile y del Centro de Microdatos. Ruiz-Tagle agradece el financiamiento proporcionado por la Iniciativa Científica Milenio al Centro de Microdatos, Proyecto P07S-023-F.

${ }^{1}$ Superintendencia de Pensiones, Chile.

${ }^{2}$ De acuerdo con la Encuesta de Protección Social (EPS), el porcentaje de pensionados pasa de un 13,3\% en 2004 a un 14,5\% en 2006.

${ }^{3}$ Ley N ${ }^{\circ} 19.943$.

${ }^{4}$ De acuerdo a la ley $N^{\circ} 19.943$ vigente, este porcentaje se ajustó al $70 \%$ el 19 de agosto de 2010. motora propia de ese período de su vida que en la adultez media. De esta manera, la forma en que valora los años que le quedan por vivir (esperanza de vida) puede generar heterogeneidad en la impaciencia relativa. La incertidumbre que rodea el poder disfrutar de calidad de vida en el futuro hará que los individuos renuentes al riesgo prefieran el consumo presente al consumo futuro. En este artículo se ofrece evidencia de que mientras más alto es el nivel de aversión al riesgo del individuo, más se exacerba su impaciencia relativa.

En el presente documento, la decisión de pensionarse será representada por medio de un modelo de utilidad agregada de dos períodos, de modo que si el imponente se pensiona al inicio del primer período, lo está haciendo de forma anticipada, mientras que si lo hace al inicio del segundo período, se está pensionando a la edad legal. Así, la edad legal condiciona el corte entre estos períodos. En la estimación se utilizó un modelo de elección discreta en que solo se distingue si el individuo se pensiona de forma anticipada o no. La estimación toma la estructura sugerida por el modelo teórico, tratando de identificar la incidencia de la percepción de la calidad de vida futura de acuerdo con su esperanza de vida (Bleichrodt y Quiggin, 1999) en la impaciencia que conduce a pensionarse anticipadamente.

Los resultados muestran que aquellos que se pensionan anticipadamente tienen una mayor tasa de descuento intertemporal, lo que se explicaría por la esperanza de vida futura. Además, se encuentra cierta evidencia de que mientras más alto es el nivel de aversión al riesgo, mayor es la impaciencia por pensionarse de forma anticipada, lo que podría deberse a la incertidumbre sobre la calidad de vida futura.

El resto del artículo se organiza de la siguiente manera: luego de esta Introducción, en la sección II se desarrolla una breve revisión del contexto de la regulación, los datos y la literatura existente. En la sección III se presenta el marco teórico y el método de estimación utilizado. La sección IV contiene el análisis empírico, la descripción de variables y los resultados empíricos, además de algunas posibles extensiones y consideraciones. Finalmente, se presentan las principales conclusiones. 


\section{II}

\section{Revisión de la literatura sobre pensiones}

Antes de 1980, en Chile existía un sistema de reparto para el pago de pensiones. Sin embargo, a partir de esa fecha se consolida una reforma estructural definitiva al sistema de pensiones ${ }^{5}$. Esta reforma permite que se cierre progresivamente el sistema de reparto existente, que es sustituido por el sistema de capitalización individual. Este mecanismo de financiamiento, a diferencia del sistema anterior, depende de la rentabilidad del mercado.

Una parte de la literatura sobre pensiones se concentra en la investigación de los factores que influyen en la decisión de adelantar la pensión. Se ha propuesto que la decisión de pensionarse de manera anticipada será mayor mientras más altos sean los beneficios de la seguridad social (Mitchell y Phillips, 2000), mientras mayores sean los ahorros adicionales con que cuentan las personas (Au, Mitchell y Phillips, 2005), y mientras peor sea la percepción de salud que estas tienen respecto de sí mismas (Hammitt, Haninger y Treich, 2005). Además, algunos trabajos se apoyan en simulaciones para explicar esta decisión de pensión. Por ejemplo, Poterba, Rauh y Venti (2005) sostienen que incluso un hogar con bajo nivel de riesgo podría alcanzar un buen margen de utilidad futura si realiza una diversificación adecuada de sus inversiones que le permitiría pensionarse de manera anticipada. Alternativamente, Diamond y Köszegi (2003) proponen un modelo hiperbólico modificado para argumentar que la falta de

\footnotetext{
${ }^{5}$ Decreto Ley $\mathrm{N}^{\circ} 3.500$.
}

autocontrol influye en la conducta de jubilación, sobre todo en anticipar la pensión.

Asimismo, la decisión de pensionarse por anticipado puede estar condicionada por las circunstancias del ciclo económico. Por una parte, un período de alto desempleo puede disminuir aún más la probabilidad de encontrar empleo para aquellos cercanos a la edad de retiro, lo que incentivaría un retiro temprano (Hairault, Langot y Sopraseuth, 2010). Por otra parte, un ciclo económico recesivo puede empujar los salarios a la baja hasta el punto de inducir una mayor probabilidad de pensión anticipada en un contexto de oferta de trabajo endógena (Chai y otros, 2009). Paralelamente, un ciclo económico recesivo puede llevar a la baja las tasas de retorno con que se calculan las pensiones futuras, haciendo menos atractiva la pensión anticipada e induciendo de esta manera un retraso en la pensión.

La percepción del propio estado de salud y la esperanza de vida están íntimamente relacionadas. French (2005) encuentra evidencia de que el estado de salud o la incertidumbre de poder mantenerla en el tiempo inciden en la decisión de pensionarse. Por otra parte, Guiso y Paiella (2006) constatan que la aversión al riesgo permite predecir una serie de decisiones en el hogar, como es la decisión de pensión anticipada. Asimismo, la tolerancia al riesgo se relaciona positivamente con la percepción de salud y la esperanza de vida (Hammitt, Haninger y Treich, 2005). De esta manera, en este trabajo se procura integrar formalmente la aversión al riesgo en la decisión de pensión anticipada, junto con la percepción de salud y de esperanza de vida, a través de la valoración de la calidad futura de vida. 


\section{III}

\section{Modelo teórico y estrategia empírica}

A continuación se desarrolla un modelo que da sustento formal a la influencia de la impaciencia y la aversión al riesgo en la decisión de pensionarse anticipadamente. Para ello se formaliza analíticamente la decisión de pensión anticipada o en edad legal mediante un modelo simple de ciclo de vida dividido en dos períodos. De este modo, si el individuo se pensiona de forma anticipada, lo hará al principio del primer período, y de hacerlo de forma retardada, lo hará al inicio del segundo período.

Diremos que $U(\cdot)$ representa la utilidad agregada bajo condiciones de separabilidad lineal, mientras que $u(\cdot)^{6}$ corresponderá a la utilidad en un período, de manera que $u^{\prime}\left(C_{t}\right)>0$ y $u^{\prime \prime}\left(C_{t}\right)<0$. Además, se asume que la función de utilidad en cada período es isoelástica (CRRA, por sus siglas en inglés), de manera que $\sigma$ representa el nivel de aversión relativa al riesgo relativo constante, $\rho$ es la tasa de descuento intertemporal, que se denotará como el factor de descuento $\beta(H, \sigma)$, que se vinculará con la aversión al riesgo $\sigma$ y la esperanza de vida $H$.

El problema de maximización de bienestar del individuo consiste en:

$$
\begin{aligned}
& \max _{C_{t}, C_{t+1}} U\left(C_{t}, C_{t+1}, \sigma, \rho, H\right)= \\
& u\left(C_{t}, \sigma\right)+\beta(H, \sigma) \cdot u\left(C_{t+1}, \sigma\right)
\end{aligned}
$$

Sujeto a: $\quad C_{t}+A_{t} \leq Y_{t}+\lambda \cdot B$

$$
C_{t+1} \leq(1+r) \cdot A_{t}+(1+s)(1-\lambda) \cdot B \quad A_{t} \geq 0
$$

donde $r$ representa la tasa de interés de mercado, mientras que $s$ es la rentabilidad ofrecida por las AFP. Por su parte, $B$ corresponde al monto acumulado de pensión, mientras que $\lambda$ es la fracción que recibe la persona en el momento de pensionarse, la que se puede ajustar dependiendo de cuán tan anticipadamente se pensione. En caso de pensionarse a la edad legal, $\lambda$ tomará el valor de cero (0). Finalmente $A_{t}$ representa el nivel de activos inicial.

Con el objeto de incorporar la valoración de la esperanza de vida y la aversión al riesgo en la impaciencia relativa, se define el factor de descuento de la forma

\footnotetext{
${ }^{6}$ Se supondrá que la función satisface las condiciones de Inada, $\lim _{C \rightarrow \infty} u^{\prime}(C)=0$ y $\lim _{C \rightarrow 0} u^{\prime}(C)=\infty$
}

$\beta(H, \sigma)=\delta \cdot \phi(H, \sigma)$. El parámetro $\delta$ es una constante que representa la distorsión en el factor de descuento debido a la diferencia en la valoración de la calidad de vida presente de cada individuo. En cuanto a la función $\phi(H, \sigma)$, se asume que para un mismo nivel de aversión al riesgo $\lim _{H \rightarrow 0} \phi(H, \sigma)=0$, lo que quiere decir que si un individuo no espera vivir un período más, su única oportunidad de disfrutar de mejor calidad de vida es en el presente. Además, se asume que $\lim _{H \rightarrow \infty} \phi(H, \sigma)=1$, lo que indica que el tiempo de calidad futura no rivaliza con el presente.

Proposición 1. La función $\phi(H, \sigma)$ se define creciente y convexa en $H, \frac{\partial \phi}{\partial H}>0$ y $\frac{\partial^{2} \phi}{\partial H^{2}}<0$. Al mismo tiempo, se define decreciente y cóncava en $\sigma, \frac{\partial \phi}{\partial \sigma}<0$ y, $\frac{\partial^{2} \phi}{\partial \sigma^{2}}<0$.

Dadas las dificultades existentes para acceder a los créditos, se supondrá que en esta economía los individuos se ven enfrentados a restricciones de liquidez.

Algunos factores mencionados en la literatura y que podrían explicar una caída en la utilidad marginal futura, son el factor no contributivo de la seguridad social, los riesgos financieros y el estado de la salud. La seguridad social y el mercado financiero no están bajo el control del individuo. No obstante, la percepción que el individuo tiene de su condición de salud le permite estimar su esperanza de vida; de hecho, Engen, Gale y Uccello (1999) muestran que no considerar este elemento genera serias limitaciones en las estimaciones de los modelos de consumo intertemporal. Alternativamente, se puede considerar que esta condición se debe a la disposición sicológica con que el individuo enfrentará los años en el futuro.

En el modelo planteado, la componente $\phi(H, \sigma)$ representa la valoración futura que el individuo tiene de su esperanza de vida, similar a la presentación de la longevidad como la probabilidad de vivir descrita en Bleichrodt y Quiggin (1999), o como la incorporación del estado salud en el factor de descuento intertemporal en Nordhaus (2002). La idea es establecer que un individuo descuenta el futuro según como crea que se encontrarán sus condiciones anímicas y físicas.

Las restricciones expresadas en el modelo de la ecuación (1) en forma conjunta se pueden resumir en:

$$
C_{t}+\frac{1}{1+r} C_{t+1} \leq Y_{t}+B+\frac{(s-r)}{(1+r)}(1-\lambda) \cdot B
$$


Por simplicidad, se denotará $R=1+r$. Se considerará que al final de su vida el individuo consume toda su riqueza. Por otra parte, si $s<r$, todos los individuos — sin importar su grado de aversión e impaciencia— se pensionarán en forma anticipada, ya que el mercado les estaría ofreciendo una mejor alternativa financiera, independientemente de lo que decidan hacer con su riqueza una vez pensionados. De esta manera, la decisión de pensionarse anticipadamente es relevante solo si la tasa de rentabilidad de la cuenta individual es mayor o igual que la del mercado ${ }^{7}$.

La condición de equilibrio del problema de la ecuación (1), se reduce a:

$$
\begin{gathered}
T M S=-(1+r)=-R \\
T M S=-\frac{\partial U / \partial C_{t}}{\partial U / \partial C_{t+1}}=-\frac{u^{\prime}\left(C_{t}, \sigma\right)}{\delta \cdot \phi(H, \sigma) \cdot u^{\prime}\left(C_{t+1}, \sigma\right)}=-R \\
\frac{u^{\prime}\left(C_{t}, \sigma\right)}{\delta \cdot \phi(H, \sigma) \cdot u^{\prime}\left(C_{t+1}, \sigma\right)}=R
\end{gathered}
$$

Paralelamente, la relación entre consumo futuro y presente debe ser menor para los individuos que deciden pensionarse en forma anticipada, $A$, que para aquellos que deciden hacerlo a la edad legal, $L$. Esto ocurre porque los primeros estarían sustituyendo menor consumo futuro por mayor consumo presente. Ello se expresa en la ecuación (3).

$$
\frac{C_{t+1}^{A}}{C_{t}^{A}}<\frac{C_{t+1}^{L}}{C_{t}^{L}}
$$

De esta manera, si la función de utilidad es isoelástica $^{8}$, de la forma $u\left(C_{t}, \sigma\right)=C_{t}^{1-\sigma} /(1-\sigma)$, y los niveles de consumo son mayores que la unidad y con un índice de aversión al riesgo $\sigma>1$, se cumple que:

\footnotetext{
${ }^{7}$ Es importante considerar que los ciclos económicos afectan tanto a la tasa de rentabilidad de la cuenta individual como a la rentabilidad de mercado, principalmente a través de un efecto nivel y pudiendo también afectar a las diferencias entre dichas tasas. No obstante, los ciclos recesivos que conducen a la caída de las tasas de retorno con las que se calculan las pensiones futuras pueden redundar en la disminución de la propensión a pensionarse tanto a la edad legal como de manera anticipada. Sin embargo, ello supone la expectativa de que las tasas de retorno futuro aumentarán en el corto plazo y no afecta a la propensión a pensionarse derivada de la diferencia de tasas de mercado y de las cuentas individuales.

${ }^{8}$ Función de utilidad de aversión relativa al riesgo constante (Constant Relative Risk Aversion - CRRA).
}

$$
\frac{u^{\prime}\left(C_{t}^{A}, \sigma\right)}{u^{\prime}\left(C_{t+1}^{A}, \sigma\right)}<\frac{u^{\prime}\left(C_{t}^{L}, \sigma\right)}{u^{\prime}\left(C_{t+1}^{L}, \sigma\right)}
$$

Sin embargo, la ecuación (2) se debe cumplir tanto para los que se pensionaron en forma anticipada como para aquellos que lo hicieron a la edad legal, es decir

$$
\begin{gathered}
\frac{u^{\prime}\left(C_{t}^{A}, \sigma\right)}{\delta \cdot \phi_{A}\left(H^{A}, \sigma^{A}\right) \cdot u^{\prime}\left(C_{t+1}^{A}, \sigma\right)}= \\
\frac{u^{\prime}\left(C_{t}^{L}, \sigma\right)}{\delta \cdot \phi_{L}\left(H^{L}, \sigma^{L}\right) \cdot u^{\prime}\left(C_{t+1}^{L}, \sigma\right)}=R
\end{gathered}
$$

Entonces se cumple que $0<\phi_{A}\left(H^{A}, \sigma^{A}\right)<\phi_{L}\left(H^{L}, \sigma^{L}\right)<1$, de lo que se desprende que aquel que adelanta su pensión es más impaciente, debiendo descontar más el futuro. Esta diferencia en el factor de descuento puede ocurrir: i) por una diferencia en la valoración de la esperanza de vida $^{9}$; ii) por diferencias en la esperanza de vida, o iii) por una diferencia en el nivel de aversión al riesgo.

Si los individuos, igualmente renuentes al riesgo $\left(\sigma^{A}=\sigma^{L}=\sigma\right)$, perciben una misma esperanza de vida $\left(H^{A}=H^{L}=H\right)$, entonces, para adelantar su pensión deben valorarla en forma diferente $\left(\phi\left(H^{A}, \sigma\right)=\phi\left(H^{L}, \sigma\right)\right)$. Siendo la esperanza de vida los años que le restan por vivir, diremos que disfrutar de una calidad de vida mejor en el presente que en el futuro equivaldrá a una menor valoración futura de la esperanza de vida (menor factor de descuento). No obstante, podría ocurrir que valorara de igual manera los años que le quedan $\left(\phi\left(H^{A}, \sigma\right)=\phi\left(H^{L}, \sigma\right)\right)$, en cuyo caso, para desear adelantar su pensión, deberá creer que vivirá menos años $\left(H^{A}<H^{L}\right)$.

Por otra parte, asumiendo la misma esperanza de vida $\left(H^{A}=H^{L}=H\right)$, dos individuos con coeficientes de aversión al riesgo relativo iguales a $\sigma_{0}$ y $\sigma_{1}$, respectivamente, serán renuentes al riesgo si $\sigma_{0}>1$ y $\sigma_{1}>1$. Sin embargo, si $\sigma_{0}<\sigma_{1}$, el primero resulta ser menos renuente al riesgo que el segundo. Así, de las ecuaciones (3) y (4) se desprende que:

$$
\frac{u^{\prime}\left(C_{t}^{j}, \sigma_{1}\right)}{u^{\prime}\left(C_{t+1}^{j}, \sigma_{1}\right)}<\frac{u^{\prime}\left(C_{t}^{j}, \sigma_{0}\right)}{u^{\prime}\left(C_{t+1}^{j}, \sigma_{0}\right)} \quad \forall j=A, L
$$

De esta manera, la nueva condición se muestra en la ecuación (7), donde los términos (b) y (c) representan la

\footnotetext{
${ }^{9}$ Esta diferencia se debería a que el grupo de pensionados anticipados apreciarían más los primeros años de esta esperanza de vida que el grupo de pensionados a la edad legal.
} 
condición de equilibrio planteada en (5), mientras que (a) representa la condición de equilibrio para un individuo que se pensiona de manera anticipada, pero que es más averso al riesgo que el definido en $(b)$.

$$
\begin{gathered}
\underbrace{\frac{u^{\prime}\left(C_{t}^{A}, \sigma_{1}\right)}{\delta \cdot \phi_{A}\left(H^{A}, \sigma_{1}\right) \cdot u^{\prime}\left(C_{t+1}^{A}, \sigma_{1}\right)}}_{(a)}=\underbrace{\frac{u^{\prime}\left(C_{t}^{A}, \sigma_{0}\right)}{\delta \cdot \phi_{A}\left(H^{A}, \sigma_{0}\right) \cdot u^{\prime}\left(C_{t+1}^{A}, \sigma_{0}\right)}}_{(b)}= \\
\underbrace{\frac{u^{\prime}\left(C_{t}^{L}, \sigma_{0}\right)}{\delta \cdot \phi_{L}\left(H^{L}, \sigma_{0}\right) \cdot u^{\prime}\left(C_{t+1}^{L}, \sigma_{0}\right)}}_{(c)}=R
\end{gathered}
$$

Ahora, para que se cumpla (7), debe ocurrir que:

$$
\begin{gathered}
0 \times \phi_{1}\left(H^{\alpha}, \sigma_{1}\right)<\phi_{\lambda}\left(H^{\alpha}, \sigma_{0}\right)< \\
\phi_{\alpha}\left(H^{2}, \sigma_{0}\right)<1
\end{gathered}
$$

De este modo, si un individuo se pensiona anticipadamente es porque descuenta más el futuro. Este menor factor de descuento se debe a la diferencia en la valoración presente de la esperanza de vida futura. Esta diferencia se verá exacerbada por una mayor aversión al riesgo, debido a la incertidumbre de poder disfrutar de una buena calidad de vida en el futuro.

\section{Estrategia empírica para identificar los determinantes de la pensión anticipada}

Si bien el modelo teórico da luces acerca del comportamiento que deberían tener los individuos respecto de la decisión de pensionarse considerando su esperanza de vida y aversión al riesgo, las hipótesis que se derivan del modelo no son medibles de manera directa en una estimación econométrica. Por ello, a continuación se desarrolla una estrategia empírica con la que se procura incorporar las características del modelo teórico de una manera simple y estimable.

La decisión de pensionarse de manera anticipada o a la edad legal se puede representar como un problema de elección discreta, donde un individuo racional optará por la acción que le reporte un mayor nivel de utilidad. En este sentido, es comúnmente aceptado considerar la utilidad indirecta en el análisis de la elección discreta, lo que internaliza las restricciones provenientes del ingreso y otras restricciones (Deaton y Muellbauer, 1980; Hensher, Barnard, Truong, 1988).

Para la aproximación empírica se considera la variable dicotómica $Y$, que representa la decisión de pensión, siendo igual a 1 si el individuo decide pensionarse de forma anticipada, lo que ocurrirá si la utilidad indirecta latente de estar pensionado antes de la edad legal, $U_{A}^{*}$, es mayor que la de pensionarse cuando se cumpla la edad legal, $U_{L}^{*}$. En caso contrario, la variable de decisión tomará el valor cero (0), tal y como se define a continuación:

$$
Y=\left\{\begin{array}{lll}
1 & \text { si } & U_{A}^{*}>U_{L}^{*} \\
0 & \text { si } & U_{A}^{*} \leq U_{L}^{*}
\end{array}\right.
$$

En la ecuación (8) se muestran las condiciones bajo las cuales un individuo decide pensionarse anticipadamente o a la edad legal, teniendo presente que uno de los parámetros que rige la función de utilidad es la aversión al riesgo.

Tomando la función de utilidad definida en la ecuación (1), se plantea una variable latente que representa a la función de utilidad indirecta para el individuo $i$ y la decisión $j$ de la forma:

$$
\begin{gathered}
U_{i j}^{*}(\cdot)=u\left(C_{i j, t}^{*}, \sigma_{i}\right)+\delta \cdot \phi_{j}\left(H_{i}, \sigma_{i}\right) \cdot u\left(C_{i j, t+1}^{*}, \sigma_{i}\right) \\
\forall i=1, \ldots, n ; j=A, L
\end{gathered}
$$

donde la tasa de descuento intertemporal se describe por $\beta(H, \sigma)=\delta \cdot \phi(H, \sigma)$, tal como se detalla en la sección III de este documento.

Se dará por sentado que ambos grupos poseen el mismo nivel de esperanza de vida. Sobre esta base, se supondrá una forma ad hoc para la valoración de la esperanza de vida igual a:

$$
\begin{gathered}
\phi_{j}\left(H_{i}, \sigma_{i}\right)=\phi\left(H_{i}, \sigma_{i} \mid a_{j}\right)=1-H_{i}^{-a_{j} / \sigma_{i}} \\
\forall i=1, \ldots, n ; j=A, L ; a_{j}>0
\end{gathered}
$$

Se considerará que ambos grupos creen que vivirán la misma cantidad de años, por ende, la diferencia en el factor de descuento vendrá dada por la diferencia en la valoración de la esperanza de vida representada por el parámetro $a_{j}$ en la ecuación (10). 
La forma funcional descrita en la ecuación (10) cumple con la Proposición 1, de manera que para un nivel de aversión al riesgo constante, su comportamiento será como el del gráfico 1 . El parámetro $a_{j}$ simboliza cuánto valora su esperanza de vida, lo que se podría interpretar como la valoración de la calidad de vida futura; entonces se cumple que: $a_{A}<a_{L}$.

En el caso de mantener la misma valoración de la esperanza de vida $H$, pero pasando de un nivel de aversión al riesgo $\sigma_{0}$ a uno más renuente al riesgo $\sigma_{1}$, siendo $\sigma_{0}<\sigma_{1}$, se obtiene una menor valoración de los años futuros por vivir. Esto se observa en el gráfico 2 .

Ahora, si se reemplaza (10) en (9), se obtiene una forma más extendida de la utilidad indirecta.

$$
\begin{gathered}
U_{i j}^{*}(\cdot)=\underbrace{u\left(C_{i j, t}^{*}, \sigma_{i}\right)+\delta \cdot u\left(C_{i j, t+1}^{*}, \sigma_{i}\right)}_{(I)}- \\
\underbrace{\delta \cdot H_{i}^{-a_{j} / \sigma_{i}} u\left(C_{i j, t+1}^{*}, \sigma_{i}\right)}_{(I I)}
\end{gathered}
$$

La componente (I) presenta el modelo clásico de descuento en dos períodos. Por su parte, la componente (II) es un término adicional de "pérdida" de utilidad por consumo futuro, debida a la valoración de la esperanza de vida presente con respecto a la del futuro.

A objeto de hacer estimable el modelo y dado que las componentes $(I)$ y (II) de la ecuación (11) se encuentran separadas aditivamente, se definirán aproximaciones para cada componente, como:

$$
\begin{gathered}
U_{i j}^{*}(\cdot)=\underbrace{\gamma_{i j} \cdot X_{i j}}_{(I I I)}+\underbrace{\alpha_{i j} \cdot h_{i}+\lambda_{i j} \cdot \sigma_{i} \cdot h_{i}}_{(I V)} \\
\forall i=1, \ldots, n ; j=A, L
\end{gathered}
$$

De esta manera, el término (III) representa la aproximación lineal tradicional al modelo de consumo intertemporal de dos períodos descrito en $(I)$. Esto corresponde al conjunto de atributos que definen las preferencias del individuo, representado por el vector $X$. Además, este establece una estructura para la función de utilidad. Este marco de trabajo permite controlar

GRÁFICO 1

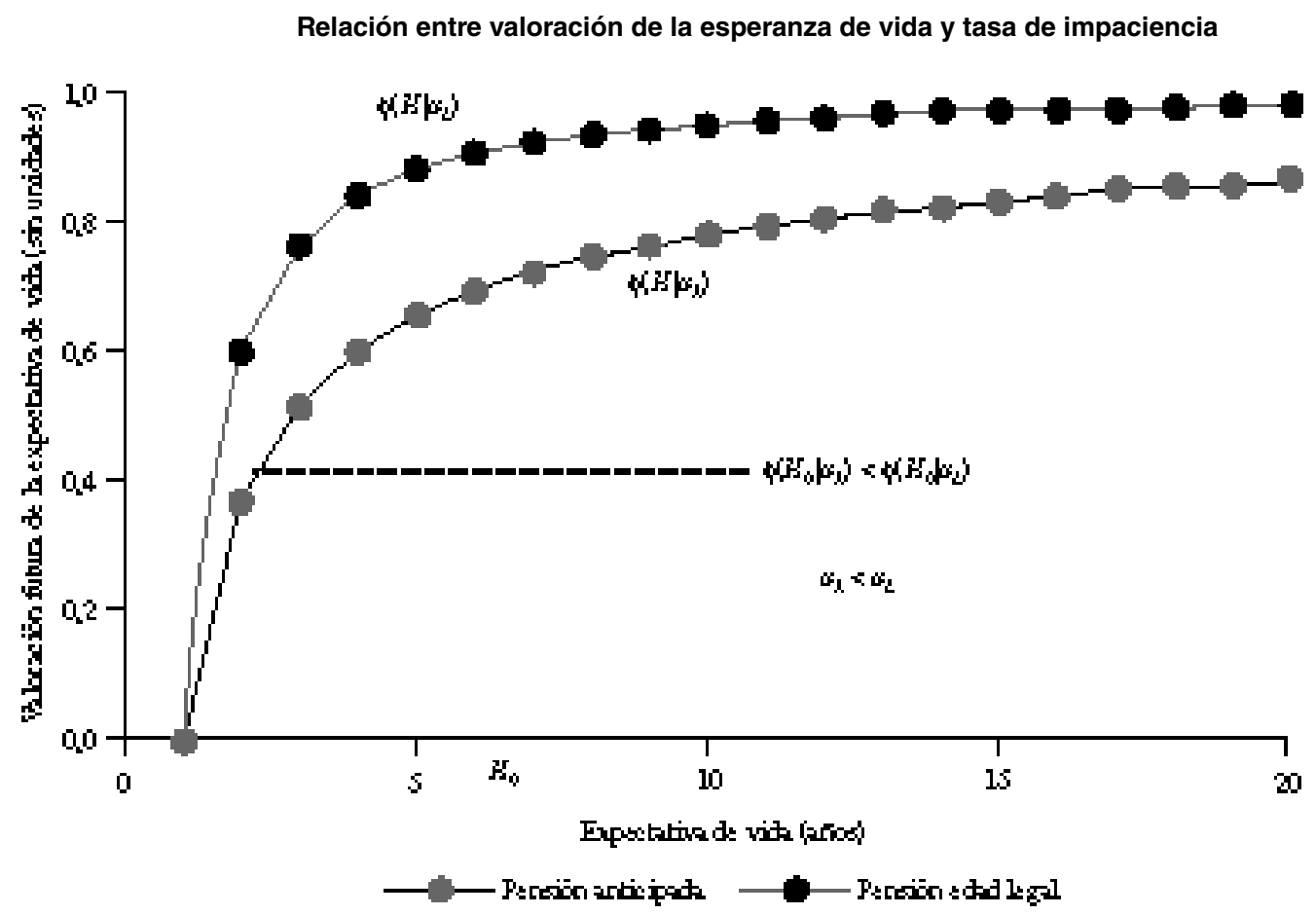

Fuente: elaboración propia. 
GRÁFICO 2

\section{Menor valoración de la esperanza de vida debido al futuro incierto}

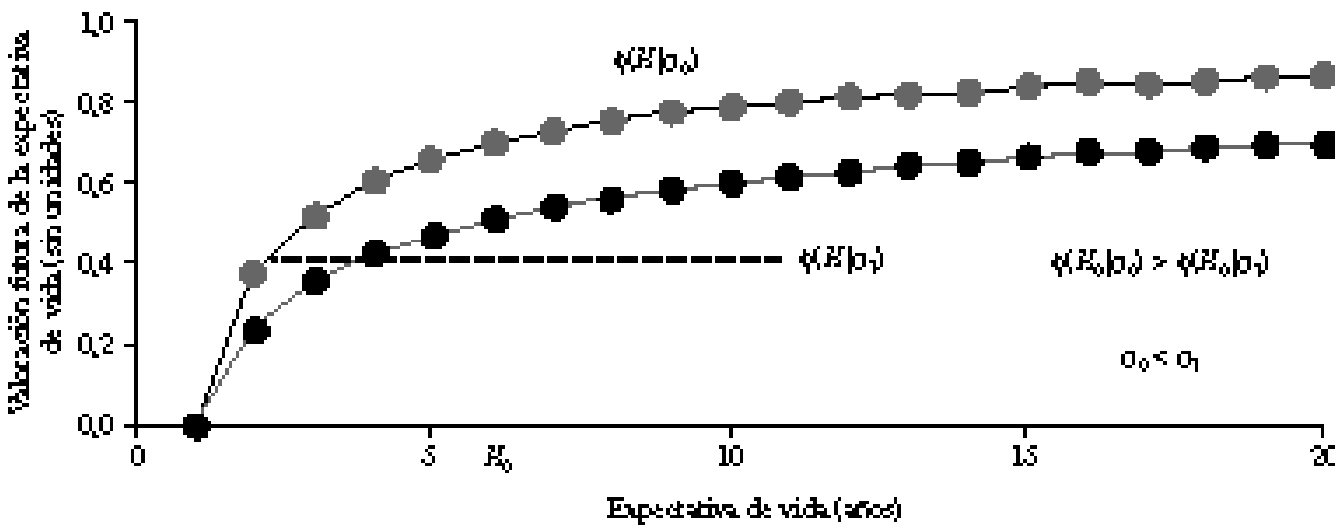

- Ameriön alritsgo medio

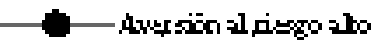

Fuente: elaboración propia.

por otras preferencias de las personas (contenidas en el vector $X$ ) que puedan afectar a la función de utilidad y, por consiguiente, a la decisión de pensionarse a través de una forma funcional lineal en un enfoque semi reducido.

La componente (II) de la ecuación (11) fue aproximada por medio de la componente $(I V)$ de la ecuación (12), con el propósito de capturar parte de la no linealidad presentada. De este modo, $h_{i}=\ln \left(H_{i}\right)$ y el parámetro $\alpha_{i j}$ representan la caída en la utilidad futura debido al mayor descuento por valorar más los primeros años de lo que le queda por vivir, término que se ve afectado tanto en magnitud como en signo por los cambios en $a_{j}$. Este debe ser negativo y mayor en valor absoluto en aquellos que adelantaron su pensión. Además, el parámetro $\lambda_{i j}$ representa la valoración de la esperanza de vida frente al riesgo futuro, dado que el individuo es averso al riesgo. Combinar la esperanza de vida y la aversión al riesgo es un recurso conveniente para ilustrar cómo la mayor aversión al riesgo exacerba la decisión de pensionarse anticipadamente. Esto debido al riesgo de no poder disfrutar de calidad de vida en el futuro, evitando así la influencia de otros riesgos, como el riesgo financiero. De este modo, la decisión de pensionarse anticipadamente se representa como una probabilidad, de tal suerte que:

$$
\begin{gathered}
\operatorname{Pr}\left(Y_{i}=1\right) \approx \operatorname{Pr}\left(U_{A}^{*}-U_{L}^{*} \approx \Gamma_{i} \cdot X_{i}\right. \\
\left.+\gamma_{i} \cdot h_{i}+\Lambda_{i} \cdot \sigma_{i} \cdot h_{i}>\varepsilon_{A}-\varepsilon_{L}\right) \\
\operatorname{Pr}\left(Y_{i}=1\right) \approx \operatorname{Pr}\left(\Gamma_{i} \cdot X_{i}+\right. \\
\left.\Omega_{i} \cdot h_{i}+\Lambda_{i} \cdot \sigma_{i} \cdot h_{i}>\varepsilon_{i}\right)
\end{gathered}
$$

donde $\Gamma_{i}=\gamma_{i}^{A}-\gamma_{i}^{L}, \Omega_{i}=\alpha_{i}^{A}-\alpha_{i}^{L}$ y $\Lambda_{i}=\lambda_{i}^{A}-\lambda_{i}^{L}$. En esta especificación binaria del modelo a estimar se puede apreciar claramente la hipótesis de trabajo. En primer lugar, dado que los individuos que se pensionan de forma anticipada tienen que haber sido relativamente más impacientes que aquellos que lo hicieron a la edad legal (asumiendo todo lo demás constante), se debe cumplir que $\Omega_{i}<0$. Del mismo modo, el efecto de la aversión al riesgo en la variabilidad de la utilidad se recoge en la hipótesis en que $\Lambda_{i}>0$, que muestra, con todo lo demás constante, que a mayor aversión al riesgo mayor será la propensión a pensionarse de manera anticipada.

\section{La pensión anticipada en Chile}

En el caso chileno, cabe destacar que no todos los individuos se pueden pensionar de manera anticipada. En efecto, aquellos que se encuentran bajo el sistema antiguo de reparto no tienen la posibilidad de pensionarse anticipadamente. Podrían acceder a este derecho si el trabajo que desempeñan fuera calificado como pesado ${ }^{10}$, o si se encontraran en el sistema previsional actual al menos unos cinco años.

A partir de 1993, los afiliados tienen la posibilidad de pensionarse antes de la edad legal ${ }^{11}$, siempre y cuando el capital acumulado en su cuenta de ahorro individual les permita obtener una pensión mensual superior al

\footnotetext{
${ }^{10}$ Comisión Ergonómica Nacional (CEN).

${ }^{11}$ El artículo 64 de la Ley 100.
} 
$110 \%$ del salario mínimo legal vigente ${ }^{12}$. En 2008 entra en vigencia la reforma previsional ${ }^{13}$, que introduce un pilar solidario que atiende a todos los chilenos que no tienen ahorros previsionales. Dicha reforma también persigue mejorar el sistema de capitalización individual, junto con incentivar las cotizaciones voluntarias para una futura pensión exitosa.

Sin embargo, los afiliados al sistema de capitalización individual actual pueden adelantar su jubilación si su monto de pensión es igual o superior al $70 \%{ }^{14} \mathrm{del}$ promedio de las remuneraciones y rentas declaradas en los últimos 10 años anteriores al mes en que se acogen a este derecho. Además, debe ser igual o superior al $150 \%$ de la pensión básica solidaria de vejez (PBSV) vigente.

En el momento de pensionarse, estos afiliados también deben decidir bajo qué modalidad lo harán: si con un retiro programado, una renta vitalicia o, eventualmente, un modo mixto. Si la persona se cambió de sistema previsional y conserva sus bonos de reconocimiento, estos pueden influir en la elección de la modalidad mediante su endoso, ya que alcanzan su mayor valor al llegar a la edad legal.

Por otra parte, si el afiliado cuenta con Ahorro Previsional Voluntario (APV), este podría influir positivamente en la decisión de pensionarse en forma anticipada, ya que aumenta la probabilidad de cumplir con los requisitos en el futuro.

El aumento de restricciones progresivas ${ }^{15}$ a la pensión anticipada podría incidir en la decisión de pensionarse anticipadamente, ya que de esperar hasta el año siguiente se podrían incumplir los requisitos para hacerlo.

Para revisar la evidencia empírica en Chile se utilizará la única fuente de información disponible que permite un análisis detallado de los pensionados y de quienes podrían pensionarse de manera anticipada: la Encuesta de Protección Social (EPS). Esta encuesta, en su versión 2006, incluye a personas de 18 años de edad en adelante, representando un total poblacional de 12.426.437 individuos, de los cuales el 50,9\% son mujeres y el $49,1 \%$ son hombres. Dentro de su situación laboral, en el momento de efectuarse la encuesta, un $12,6 \%$ de la población declara encontrarse cesante,

\footnotetext{
${ }^{12}$ Estos porcentajes fueron modificados por la Ley $N^{\circ} 19.943$ que entró en vigencia en agosto del año 2004.

${ }_{13}$ Decreto Ley $\mathrm{N}^{\circ} 20.255$.

${ }^{14}$ Este porcentaje rige a partir de agosto de 2010. Durante el período comprendido entre agosto de 2006 y agosto de 2007 es del $58 \%$. Más detalles en la Superintendencia de Pensiones de Chile, http://safp.cl ${ }^{15}$ Ley No 19.943 .
}

el $57 \%$ dice estar trabajando, mientras que el $30,4 \%$ restante está inactivo ${ }^{16}$.

Sobre la base de la Encuesta de Protección Social de 2006, se puede apreciar que el ingreso monetario promedio comienza a decaer desde los 50 años, tal como se observa en el gráfico 3. Este perfil decreciente se encuentra justo en la vecindad de la edad a partir de la cual los individuos comienzan a decidir pensionarse.

Por otra parte, el número de individuos que se han pensionado de manera anticipada ha fluctuado en el tiempo (véase el gráfico 4). No obstante, se aprecia cierta relación con la rentabilidad de los fondos, que cuando es menor influiría en una menor pensión anticipada, aunque este efecto se aprecia con cierto rezago. El efecto de la crisis financiera de 2008 se refleja claramente en la rentabilidad de los fondos y en la pensión anticipada, que recién hacia 2010 recupera los niveles previos a la crisis.

Paralelamente, las estadísticas del Instituto Nacional de Estadísticas (INE) revelan que el grupo potencial de pensionados aumenta sustancialmente, hasta el punto que podría llegar a ser del orden del $20 \%$ de la población en el año 2016.

En el año 2006, un 9,2\% de los individuos "autorreportan" haberse pensionado por algún motivo, de los cuales poco más de la quinta parte $(20,7 \%)$ declaran haberlo hecho por invalidez. Para este último grupo la decisión de pensión es exógena, por lo que sus integrantes no son considerados en el análisis que sigue.

Al comparar el motivo de pensión con la fecha de retiro autorreportada se encuentran ciertas diferencias. En el cuadro 1 se observa que poco menos de la mitad que reportó haberse pensionado por vejez, lo hizo en forma anticipada. Al corregir el motivo de pensión por la edad legal autorreportada, el porcentaje de todos los pensionados de forma anticipada es de $35,1 \%$.

En Chile, el 94\% de la población se encuentra en el sistema de AFP, sin embargo, de los pensionados solo el $46 \%$ pertenecen a dicho sistema, el resto se encuentran

\footnotetext{
${ }^{16}$ La manera en que se pregunta por la situación ocupacional de los individuos en la EPs difiere de las encuestas de empleo (Instituto Nacional de Estadísticas (INE) o de la Encuesta de Ocupación de la Universidad de Chile). Mientras en la EPS es el individuo quien se autocalifica, en las encuestas de empleo es el analista quien clasifica. Esto redunda en que en la EPS se obtenga un gran número de individuos "autorreportados" como cesantes, en circunstancias que muchos de ellos habrían sido calificados como inactivos en las encuestas de empleo. Esto deriva en una sobreestimación de la tasa de desocupación y de la tasa de participación laboral, efecto sobre todo importante en el caso de las mujeres.
} 
GRÁFICO 3 Ingreso monetario promedio por edad (En miles de pesos)

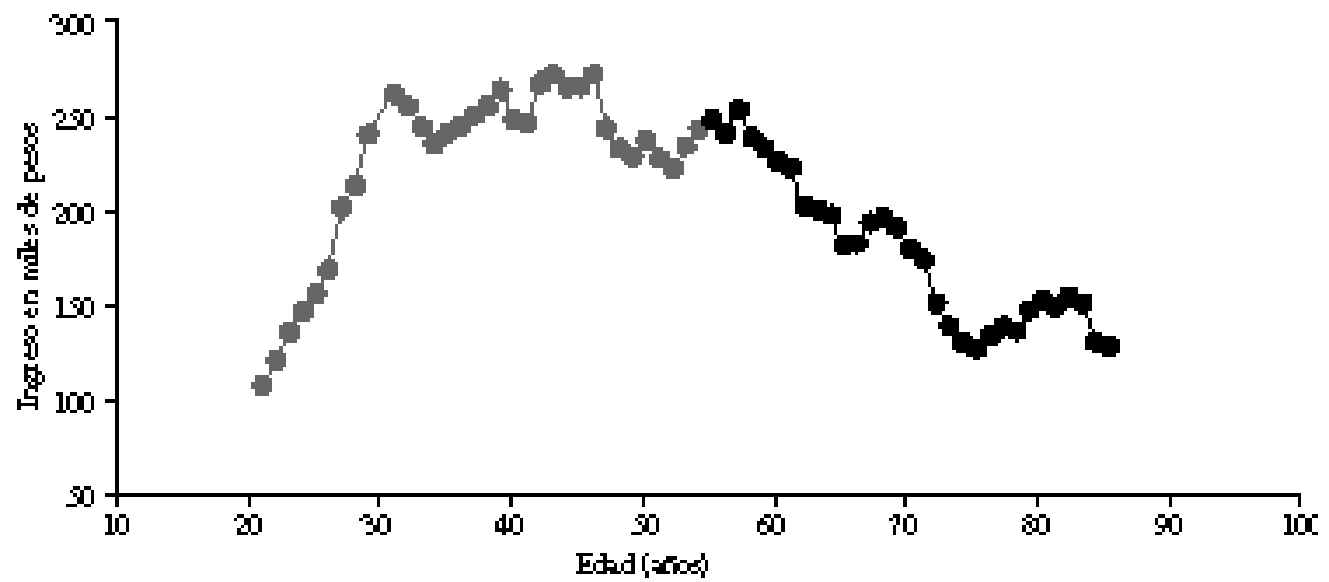

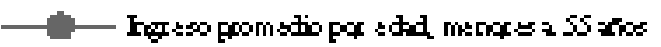

Fuente: elaboración propia sobre la base de la Encuesta de Protección Social (EPS) de 2006.

GRÁFICO 4

Pensionados por trimestre con la rentabilidad promedio de las AFP entre 2004 y 2010

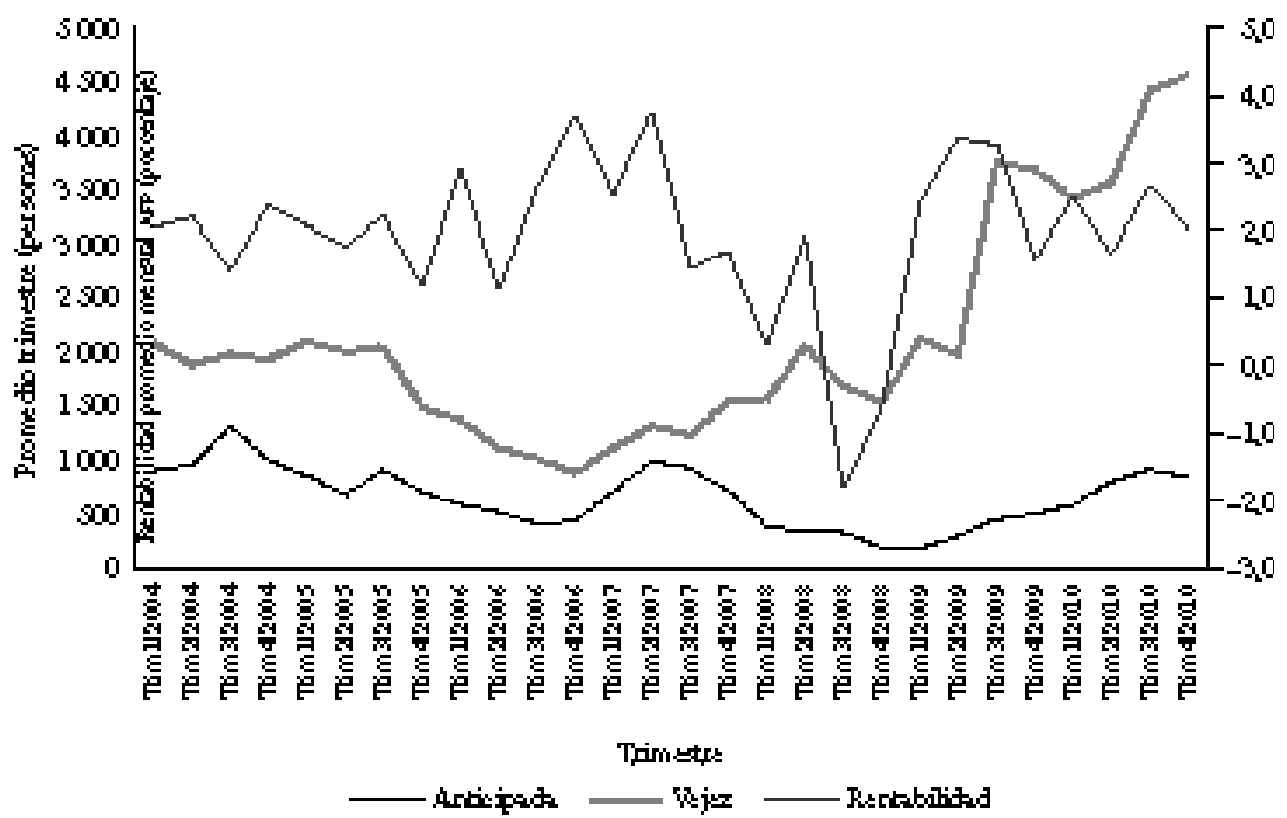

Fuente: elaboración propia sobre la base de Superintendencia de Pensiones de Chile.

AFP: administradoras de fondos de pensiones. 
CUADRO 1

Tipo de pensión declarada por los encuestados

\begin{tabular}{lccrr}
\hline \multirow{2}{*}{$\begin{array}{l}\text { Fecha de pensión } \\
\text { autorreportada }\end{array}$} & \multicolumn{2}{c}{$\begin{array}{c}\text { Motivo de pensión autorreportada } \\
\text { (en porcentajes) }\end{array}$} & Total \\
\cline { 2 - 4 } & Vejez & Anticipado & Invalidez & \\
\hline Edad legal o superior & 56,5 & 3,7 & 8,7 & 35,1 \\
Antes de la edad legal & 43,5 & 96,3 & 91,3 & 64,9 \\
Total & 100,0 & 100,0 & 100,0 & 100,0 \\
\hline
\end{tabular}

Fuente: elaboración propia sobre la base de la Encuesta de Protección Social (EPS) de 2006. Población: 1.129.325; $\mathrm{N}^{\circ}$ de observaciones: 2.375 (total que responde).

en el Instituto de Previsión Social, ex Instituto de Normalización Previsional (INP) u otro sistema ${ }^{17}$.

En la Encuesta de Protección Social (EPS) del año 2006 se incluye un conjunto de preguntas que permiten determinar cuán averso al riesgo es un individuo ${ }^{18}$. Con esta información se puede agrupar a los entrevistados en cuatro niveles de aversión al riesgo, que van de un nivel bajo (1) a un nivel alto de aversión (4) ${ }^{19}$. Esta variable de aversión al riesgo se construye luego de exponer al encuestado a la pregunta: "Suponga que Ud., como única fuente de ingresos de su hogar, debe elegir entre los siguientes dos trabajos...", donde el primer trabajo le garantiza un nivel de ingreso fijo $\mathrm{y}$ estable durante toda la vida; en el segundo trabajo tiene la misma posibilidad de ganar el doble o solo un cuarto, la mitad o tres cuartos de sus ingresos durante toda la vida.

Este esquema indagatorio de la aversión al riesgo es idéntico al que se utiliza en el Estudio de la Salud y la Jubilación (HRS, por sus siglas en inglés) de los Estados Unidos, y en la Encuesta de Ingresos y Riqueza de las Familias (shiw, por sus siglas en inglés) del Banco de Italia. Tanto en la Encuesta de Protección Social como en el HRS y la SHIW se establece una convergencia hacia la neutralidad al riesgo que podría alcanzar el encuestado, puesto que cada pregunta está dominada estocásticamente por la anterior, lo que indica que si un individuo prefiere la alternativa con menor

\footnotetext{
${ }^{17}$ De los entrevistados, menos del 3\% no reportan el motivo por el que se pensionaron, y aún menos declaran poseer Ahorro Previsional Voluntario (APV).

${ }^{18}$ Estas preguntas se encuentran en el módulo J (de la j1_1 a la j1_3).

${ }^{19} \mathrm{Se}$ debe tener presente que dentro de este módulo las preguntas son de carácter discreto y que en los niveles de aversión las preguntas no llegan a cubrir a los neutrales y amantes del riesgo.
}

CUADRO 2

\section{Niveles de aversión entre pensionados y no pensionados}

\begin{tabular}{|c|c|c|c|}
\hline \multirow{2}{*}{$\begin{array}{l}\text { Nivel de aversión } \\
\text { Mayores de } \\
18 \text { años }\end{array}$} & \multicolumn{2}{|c|}{ Pensionado } & \multirow[b]{2}{*}{ Población } \\
\hline & $\begin{array}{c}\text { No } \\
\text { (en porcentajes) }\end{array}$ & $\begin{array}{c}\text { Sí } \\
\text { (en porcentajes) }\end{array}$ & \\
\hline 1 (Bajo) & 20,3 & 14,0 & 19,8 \\
\hline 2 (Medio bajo) & 8,6 & 8,5 & 8,6 \\
\hline 3 (Medio alto) & 6,7 & 4,5 & 6,5 \\
\hline 4 (Alto) & 64,4 & 73,0 & 65,1 \\
\hline Total & 100,0 & 100,0 & 100,0 \\
\hline
\end{tabular}

Fuente: elaboración propia sobre la base de la Encuesta de Protección Social (EPS) de 2006. Población: 11.492.732; $\mathrm{N}^{\mathrm{o}}$ de observaciones: 15.052 (total que responde).

valor esperado, también preferirá aquellas con mayor valor esperado, generando un orden condicional en la selección. La distribución en porcentaje de los distintos grados de aversión al riesgo en la población de 18 años de edad y más en la Encuesta de Protección Social de 2006 se describe en el cuadro 2.

La distribución en los distintos niveles de aversión al riesgo es algo distinta entre los pensionados y no pensionados. En efecto, en el cuadro 3 se observa que la mayor diferencia se presenta en el nivel alto de aversión al riesgo, que además corresponde al de mayor concentración. Esto podría explicarse por la censura que existe respecto de los niveles superiores de aversión al riesgo.

Sin embargo, si se compara a los pensionados anticipados con los en edad legal de acuerdo con su fecha de retiro autorreportada se puede constatar que las diferencias desaparecen (véase el cuadro 3). Esto indicaría que la decisión de anticipar o no la pensión es independiente del nivel de aversión al riesgo que tiene una persona.

Por otra parte, la condición de salud sí parece jugar un papel importante en la decisión de pensionarse. En efecto, según la EPs de 2006 el motivo principal para pensionarse fueron problemas de salud en el 21,5\% de los pensionados (véase el cuadro A.3 del anexo A), seguido de un $24,3 \%$ que indicaron que se pensionaron por haber cumplido la edad legal. Asimismo, el principal motivo reportado para no seguir trabajando correspondió, en casi el $60 \%$ de los casos, a que "su salud se lo impide".

Esto nos muestra que la percepción de salud es un componente importante en la decisión de pensión. No obstante, dicha percepción se correlaciona estrechamente con la esperanza de vida, de manera que a mejor estado 
CUADRO 3

Participación de la aversión al riesgo, según pensión anticipada y edad legal

\begin{tabular}{lccr}
\hline \multirow{2}{*}{$\begin{array}{l}\text { Nivel de } \\
\text { aversión }\end{array}$ pensionados } & No & Ponsión anticipada \\
\cline { 2 - 3 } & (en porcentajes) & $\begin{array}{c}\text { Sí } \\
\text { (en porcentajes) }\end{array}$ & \\
\hline 1 (Bajo) & 15,0 & 13,2 & 13,9 \\
2 (Medio bajo) & 8,0 & 8,8 & 8,5 \\
3 (Medio alto) & 5,4 & 4,2 & 4,6 \\
4 (Alto) & 71,6 & 73,8 & 73,0 \\
& 100,0 & 100,0 & 100,0 \\
\hline
\end{tabular}

Fuente: elaboración propia sobre la base de la Encuesta de Protección Social (EPS) de 2006. $\mathrm{N}^{\circ}$ de observaciones: 1.875 (total que responde).

a Según fecha autorreportada. de salud mayor será la esperanza de vida, tal como se observa en el cuadro 4.

Otro aspecto relevante de las condiciones bajo las cuales los individuos toman sus decisiones con respecto a las pensiones es la esperanza de vida. Naturalmente, esta esperanza de vida decrece con la edad. En el gráfico 5 se aprecia que la esperanza de vida media, medida como la estimación de los años que le restan por vivir al individuo, decae según este avanza en edad, con un perfil muy similar para hombres y mujeres.

Más en particular, el interés se centra en la esperanza de vida para los individuos que se pensionan a la edad legal y aquellos que lo hacen de forma anticipada. En el gráfico 6 se denota que las tendencias son similares para ambos grupos y estas, a su vez, similares para el resto de la población.

CUADRO 4

Esperanza de vida promedio en años por nivel de percepción de salud

\begin{tabular}{|c|c|c|c|c|c|c|}
\hline \multirow{3}{*}{$\begin{array}{l}\text { Percepción del } \\
\text { estado de salud }\end{array}$} & \multicolumn{6}{|c|}{ Esperanza de vida (años) } \\
\hline & \multicolumn{2}{|c|}{ Población } & \multicolumn{2}{|c|}{ Mujer $(51,3 \%)$} & \multicolumn{2}{|c|}{ Hombre $(48,7 \%)$} \\
\hline & Porcentaje & Promedio & Porcentaje & Promedio & Porcentaje & Promedio \\
\hline Muy mala & 1,0 & 12,05 & 1,5 & 11,53 & 0,5 & 13,69 \\
\hline Mala & 5,8 & 18,25 & 7,3 & 17,80 & 4,2 & 19,09 \\
\hline Regular & 22,4 & 26,42 & 25,0 & 26,35 & 19,5 & 26,50 \\
\hline Buena & 48,8 & 38,68 & 47,7 & 38,03 & 50,1 & 39,33 \\
\hline Muy buena & 13,3 & 44,13 & 12,1 & 43,53 & 14,6 & 44,66 \\
\hline \multirow[t]{2}{*}{ Excelente } & 8,7 & 45,92 & 6,4 & 44,48 & 11,1 & 46,78 \\
\hline & 100,0 & 35,8 & 100,0 & 34,3 & 100,0 & 37,5 \\
\hline
\end{tabular}

Fuente: elaboración propia sobre la base de la Encuesta de Protección Social (EPS) de 2006. Población: 9.953.561; No de observaciones: 13.086 (total que responde).

GRÁFICO 5

Esperanza de vida promedio según edad del encuestado, por hombre y mujer

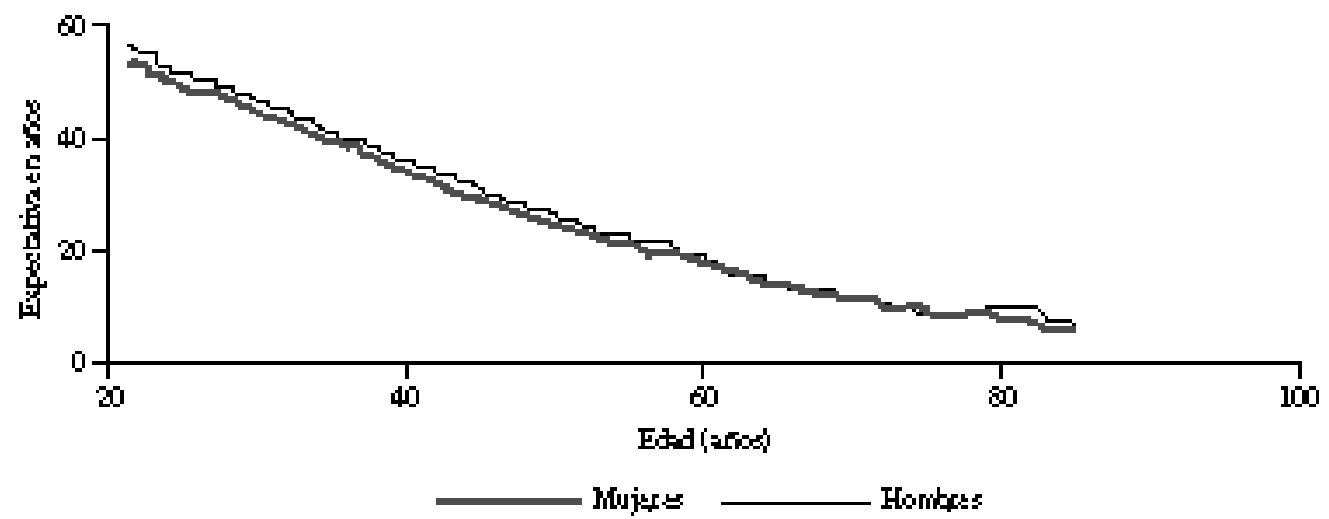

Fuente: elaboración propia sobre la base de la Encuesta de Protección Social (EPS) de 2006. 
GRÁFICO 6

Esperanza de vida promedio según edad del encuestado por estado de pensión para los hombres

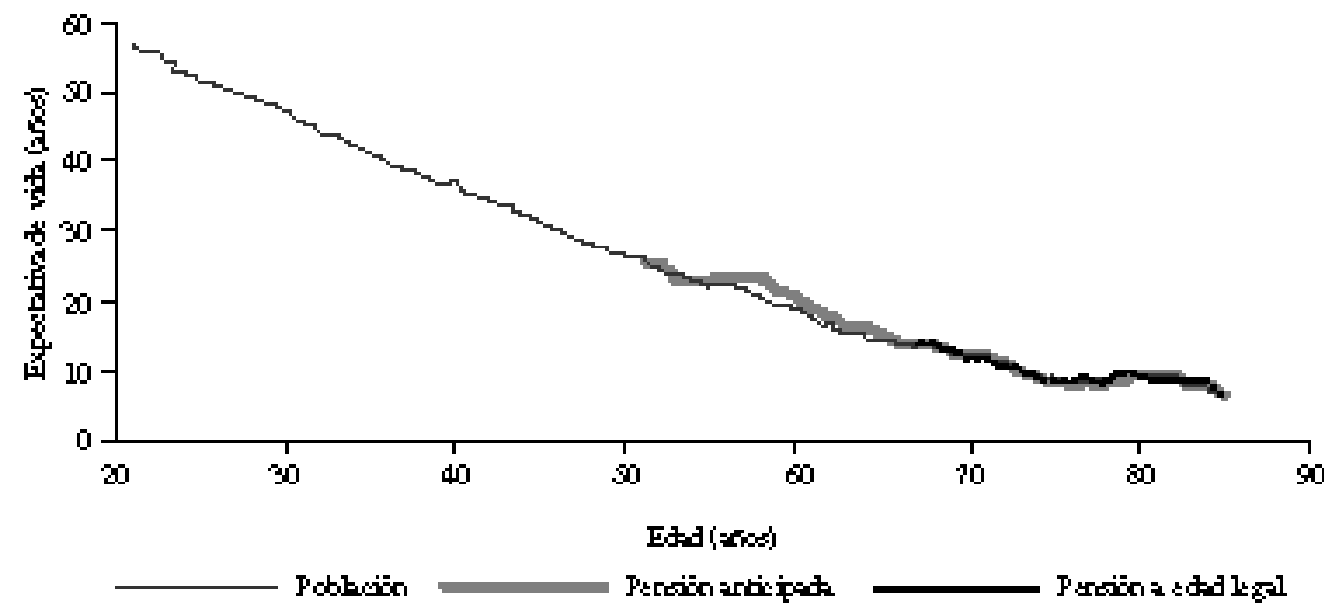

Fuente: elaboración propia sobre la base de la Encuesta de Protección Social (EPS) de 2006.

\section{Estimación de los determinantes de la pensión anticipada}

Antes de presentar los resultados de las estimaciones, es necesario revisar aspectos relacionados con la muestra y las variables que se utilizarán. Se constata que la muestra se reduce de acuerdo con una serie de consideraciones necesarias para llevar a cabo las estimaciones. En primer lugar, cabe considerar que las condiciones que exige la ley chilena para pensionarse en forma anticipada ${ }^{20}$, lo observable de esta práctica, y que los entrevistados hayan respondido al cuestionario requerido, son restricciones

${ }^{20}$ Ley No 19.943 . que limitan el número de observaciones ${ }^{21}$. Además, se definió un rango de edad de 60 a 65 años para las mujeres y de 65 a 70 años para los hombres.

Las variables que se emplean tienen que ver con las características que determinan la función de utilidad. En este caso, se define un vector de variables compuesto por características del individuo, del hogar y el mercado laboral en conjunto con el de las pensiones. Este conjunto de variables se detallan en el cuadro 5 .

La aversión al riesgo es una característica intrínseca del individuo, por lo que se define como un parámetro cuya influencia en la decisión de pensionarse antes de la

${ }^{21}$ La cantidad a nivel poblacional de la EPS de 2006 es de 12.426 .437 individuos, expandidos a partir de 16.443 encuestados, de los cuales el $8,5 \%$ no responde a la pregunta vinculada a la aversión al riesgo.

CUADRO 5

Definición del grupo de variables a considerar

\begin{tabular}{lll}
\hline Características del individuo & Características del hogar & Otras \\
\hline Género & Jefe del hogar & Conocimiento del sistema de AFP \\
Edad & Estado civil & Conocimiento sistema previsional \\
Escolaridad & Número de hijos & Conocimiento financiero \\
Percepción del estado de salud & Número de nietos & Regiones por zona \\
Percepción de la esperanza de vida & Ingreso monetario & Años en el mercado laboral \\
Nivel de aversión al riesgo & Patrimonio & Edad de inicio laboral \\
& Propiedad del domicilio & Activo laboralmente \\
\hline
\end{tabular}

Fuente: elaboración propia. 
edad legal se busca establecer. La poca continuidad en la variable de aversión al riesgo obligó a agruparla en dos niveles: el nivel alto, que concentra un $73 \%$ aproximadamente, y el nivel medio alto, medio bajo y bajo con un $27 \%$ (véase el cuadro 3 ).

La información que ayude a predecir la incertidumbre que encierra algún evento influirá en la acción que tomará un renuente al riesgo. Por ello, se incluyó el conocimiento que se tiene del mercado financiero y de las pensiones.

Se incluyó, además, un control por zonas geográficas, donde se definió la zona norte: regiones I a la IV; zona centro: regiones V a VII, y zona sur: regiones VIII a XII. De este modo, la comparación se lleva a cabo con respecto a la Región Metropolitana.

La estadística descriptiva de las variables a utilizar en las estimaciones se presenta en el cuadro 6. Se puede observar que si se considera la edad promedio que tienen los entrevistados, más su esperanza de vida futura, se obtiene que tanto el grupo de los pensionados a la edad legal, como el de los pensionados de forma anticipada, creen que su esperanza de vida es de casi 80 años.

En paralelo, dentro de la estadística descriptiva se puede destacar que los pensionados de forma anticipada son en su mayoría hombres (véase el cuadro 6). Además, los que se pensionaron antes de la edad legal reciben ingresos monetarios más bajos, lo que podría relacionarse con el hecho de que el porcentaje de los que se encuentran activos laboralmente también es menor, junto con un menor nivel promedio de escolaridad.

Los pensionados en la muestra se encuentran en el sistema previsional de AFP. Asimismo, el conocimiento del sistema de AFP y de la modalidad de pensión es levemente menor en aquellos que se pensionaron a la edad legal. Sin embargo, el conocimiento financiero resulta ser similar entre ambos grupos de pensionados (véase el cuadro 6).

\section{a) Resultados de las estimaciones}

Para estimar el modelo propuesto en la ecuación (13), se supondrá que los errores siguen una distribución normal utilizando un modelo de desviación equivalente normal (probit). Los resultados con distintas especificaciones se presentan en el cuadro 7.

Las estimaciones de los efectos marginales del modelo presentado en el cuadro 7 se observan en el cuadro 8 .

CUADRO 6

$$
\begin{aligned}
& \text { Características de los pensionados a la edad legal y de los anticipados en la muestra } \\
& \text { (En porcentajes) }
\end{aligned}
$$

\begin{tabular}{|c|c|c|c|c|c|c|}
\hline \multirow[t]{2}{*}{ Población: 134934 I Muestra: 314 obs. } & \multicolumn{2}{|c|}{ Pensionados } & \multicolumn{2}{|c|}{ Pensionado mujer [38\%] } & \multicolumn{2}{|c|}{ Pensionado hombre $[62 \%]$} \\
\hline & $\begin{array}{l}\text { Edad legal } \\
\qquad(34 \%)\end{array}$ & $\begin{array}{c}\text { Anticipado } \\
(66 \%)\end{array}$ & $\begin{array}{l}\text { Edad legal } \\
(54 \%)\end{array}$ & $\begin{array}{l}\text { Anticipado } \\
(46 \%)\end{array}$ & $\begin{array}{l}\text { Edad legal } \\
\quad(22 \%)\end{array}$ & $\begin{array}{c}\text { Anticipado } \\
(78 \%)\end{array}$ \\
\hline Género (hombre = 1) & 0,40 & 0,73 & & & & \\
\hline Escolaridad (años) & 7,1 & 8,1 & 7,9 & 10,9 & 6,0 & 7,1 \\
\hline Edad (años) & 65,3 & 66,1 & 63,4 & 62,4 & 68,1 & 67,4 \\
\hline Percepción de la salud (mala = 1 a Excelente $=6$ ) & 3,4 & 3,4 & 3,3 & 3,4 & 3,5 & 3,4 \\
\hline Percepción esperanza de vida (años) & 14,7 & 12,4 & 16,5 & 14,9 & 11,9 & 11,5 \\
\hline Nivel de aversión (alta $=1)$ & 0,7 & 0,8 & 0,8 & 0,8 & 0,7 & 0,8 \\
\hline Jefe de hogar $($ sí = 1) & 0,72 & 0,91 & 0,56 & 0,80 & 0,95 & 0,95 \\
\hline Estado civil (en pareja = 1) & 0,48 & 0,64 & 0,43 & 0,18 & 0,54 & 0,81 \\
\hline Número de hijos & 0,22 & 0,36 & 0,32 & 0,42 & 0,08 & 0,34 \\
\hline Número de nietos & 2,1 & 2,2 & 2,0 & 1,6 & 2,3 & 2,4 \\
\hline Ingreso monetario (M\$ / 2006) & 287 & 270 & 333 & 238 & 217 & 282 \\
\hline Patrimonio activos (MM\$ / 2006) & 19,85 & 25,05 & 21,88 & 20,02 & 16,75 & 26,90 \\
\hline Patrimonio deudas (MM\$ / 2006) & 0,54 & 0,86 & 0,84 & 1,87 & 0,08 & 0,49 \\
\hline Propietario de la vivienda (sí = 1) & 0,87 & 0,88 & 0,88 & 0,81 & 0,87 & 0,91 \\
\hline Conocimiento de AFP $(<\%>)$ & 0,35 & 0,40 & 0,37 & 0,45 & 0,31 & 0,38 \\
\hline Conocimiento sistema previsional $(<\%>)$ & 0,29 & 0,40 & 0,29 & 0,36 & 0,28 & 0,42 \\
\hline Conocimiento financiero $(s i ́=1)$ & 0,06 & 0,05 & 0,06 & 0,05 & 0,06 & 0,06 \\
\hline Zona norte & 0,11 & 0,12 & 0,15 & 0,11 & 0,05 & 0,13 \\
\hline Zona centro & 0,30 & 0,17 & 0,24 & 0,13 & 0,38 & 0,19 \\
\hline Zona sur & 0,19 & 0,17 & 0,13 & 0,10 & 0,29 & 0,20 \\
\hline Años en el mercado laboral & 43,3 & 39,4 & 40,1 & 33,9 & 48,1 & 41,5 \\
\hline Edad de inicio en el mercado laboral & 19,0 & 17,4 & 19,9 & 21,2 & 17,5 & 16,1 \\
\hline Activo laboralmente $($ sí $=1)$ & 0,35 & 0,43 & 0,35 & 0,33 & 0,36 & 0,46 \\
\hline
\end{tabular}

Fuente: elaboración propia sobre la base de la Encuesta de Protección Social (EPS) de 2006.

Nota: $\mathrm{M} \$=$ miles de pesos; $\mathrm{MM} \$=$ millones de pesos.

Hombres de 65 a 70 años; mujeres de 60 a 65 años. 
CUADRO 7

Estimación probit ${ }^{\mathrm{a}}$ con esperanza de vida

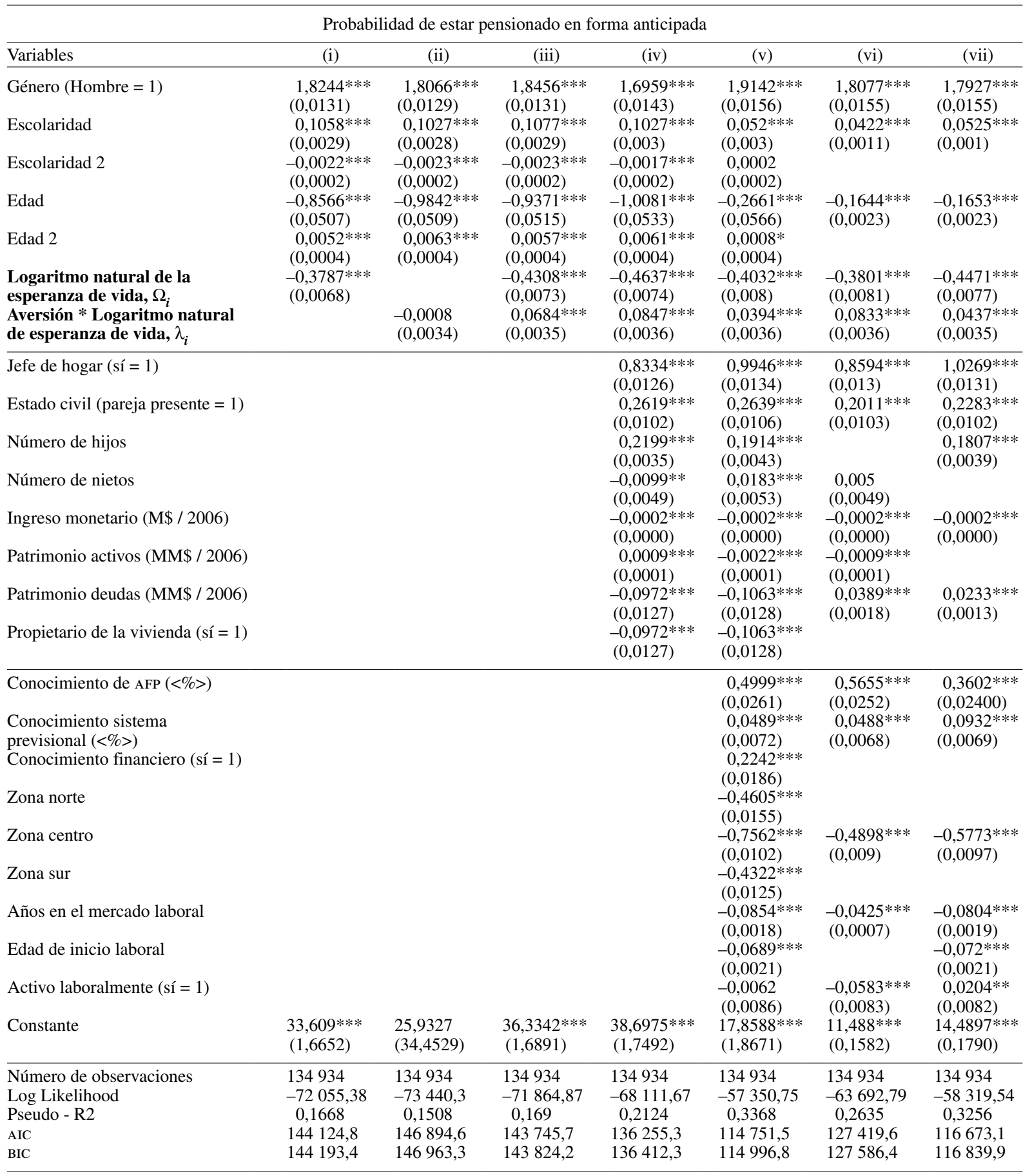

Fuente: elaboración propia sobre la base de la Encuesta de Protección Social (EPS) de 2006.

Nota: M\$ = miles de pesos; MM\$ = millones de pesos; AIC = Akaike Information Criteria; BIC = Bayesian Information Criteria.

a desviación equivalente normal.

Significativo al $* 10 \%$; ** Significativo al 5\%; *** Significativo al 1\% (desviación estándar muestral). 
CUADRO 8

Estimación de los efectos marginales para variados modelos

\begin{tabular}{|c|c|c|c|c|c|c|c|}
\hline \multicolumn{8}{|c|}{ Efectos marginales de la probabilidad de adelantar la pensión } \\
\hline Variables & (i) & (ii) & (iii) & (iv) & (v) & (vi) & (vii) \\
\hline Género (hombre = 1) & $\begin{array}{l}0,6213 * * * \\
(0,0036)\end{array}$ & $\begin{array}{l}0,6176^{* * * *} \\
(0,0036)\end{array}$ & $\begin{array}{l}0,6269 * * * \\
(0,0036)\end{array}$ & $\begin{array}{l}0,5815^{* * * *} \\
(0,0041)\end{array}$ & $\begin{array}{l}0,6181 * * * \\
(0,0041)\end{array}$ & $\begin{array}{l}0,608 * * * \\
(0,0042)\end{array}$ & $\begin{array}{l}0,5878 * * * \\
(0,0042)\end{array}$ \\
\hline Escolaridad & $\begin{array}{l}0,0376^{* * * *} \\
(0,001)\end{array}$ & $\begin{array}{l}0,0367 * * * \\
(0,001)\end{array}$ & $\begin{array}{l}0,0382^{* * * *} \\
(0,001)\end{array}$ & $\begin{array}{l}0,036 * * * \\
(0,001)\end{array}$ & $\begin{array}{l}0,0167 * * * \\
(0,001)\end{array}$ & $\begin{array}{l}0,0145^{* * * *} \\
(0,0004)\end{array}$ & $\begin{array}{l}0,0171 * * * \\
(0,0003)\end{array}$ \\
\hline Escolaridad 2 & $\begin{array}{l}-0,0008 * * * \\
(0,0001)\end{array}$ & $\begin{array}{l}-0,0008 * * * \\
(0,0001)\end{array}$ & $\begin{array}{l}-0,0008 * * * \\
(0,0001)\end{array}$ & $\begin{array}{l}-0,0006^{* * * *} \\
(0,0001)\end{array}$ & $\begin{array}{c}0,0001 \\
(0,0001)\end{array}$ & & \\
\hline Edad & $\begin{array}{l}-0,3043 \text { *** } \\
(0,0181)\end{array}$ & $\begin{array}{l}-0,3513 \text { *** } \\
(0,0182)\end{array}$ & $\begin{array}{l}-0,3326^{* * * *} \\
(0,0183)\end{array}$ & $\begin{array}{l}-0,3538 * * * \\
(0,0188)\end{array}$ & $\begin{array}{l}-0,0857 * * * \\
(0,0182)\end{array}$ & $\begin{array}{l}-0,0566^{* * * *} \\
(0,0008)\end{array}$ & $\begin{array}{l}-0,0539 * * * \\
(0,0008)\end{array}$ \\
\hline Edad 2 & $\begin{array}{l}0,0018 * * * \\
(0,0001)\end{array}$ & $\begin{array}{l}0,0023 \text { *** } \\
(0,0001)\end{array}$ & $\begin{array}{l}0,002 * * * \\
(0,0001)\end{array}$ & $\begin{array}{l}0,0022 \text { *** } \\
(0,0001)\end{array}$ & $\begin{array}{c}0,0003^{*} \\
(0,0001)\end{array}$ & & \\
\hline $\begin{array}{l}\text { Logaritmo natural de la } \\
\text { esperanza de vida, } \Omega_{i} \\
\text { Aversión * Logaritmo natural } \\
\text { de esperanza de vida, } \lambda_{i}\end{array}$ & $\begin{array}{l}-0,1345^{* * *} \\
(0,0024)\end{array}$ & $\begin{array}{c}-0,0003 \\
(0,0012)\end{array}$ & $\begin{array}{l}-0,1529 * * * \\
(0,0026) \\
0,0243 * * * \\
(0,0012)\end{array}$ & $\begin{array}{l}-0,1627 * * * \\
(0,0026) \\
0,0297 * * * \\
(0,0013)\end{array}$ & $\begin{array}{l}-0,1298 * * * \\
(0,0026) \\
0,0127 * * * \\
(0,0012)\end{array}$ & $\begin{array}{l}-0,1308 * * * \\
(0,0028) \\
0,0286 * * * \\
(0,0012)\end{array}$ & $\begin{array}{l}-0,1457 * * * \\
(0,0026) \\
0,0142 * * * \\
(0,0011)\end{array}$ \\
\hline Jefe de hogar (sí = 1) & & & & $\begin{array}{l}0,3159^{* * * *} \\
(0,0048)\end{array}$ & $\begin{array}{l}0,3643^{* * * *} \\
(0,0051)\end{array}$ & $\begin{array}{l}0,3231 * * * \\
(0,0049)\end{array}$ & $\begin{array}{l}0,3782 * * * \\
(0,005)\end{array}$ \\
\hline Estado civil (pareja presente $=1$ ) & & & & $\begin{array}{l}0,0927 * * * \\
(0,0036)\end{array}$ & $\begin{array}{l}0,086^{* * * *} \\
(0,0035)\end{array}$ & $\begin{array}{l}0,0698 * * * \\
(0,0036)\end{array}$ & $\begin{array}{l}0,0752 * * * \\
(0,0034)\end{array}$ \\
\hline Número de hijos & & & & $\begin{array}{l}0,0772 \text { *** } \\
(0,0012)\end{array}$ & $\begin{array}{l}0,0616^{* * *} \\
(0,0014)\end{array}$ & & $\begin{array}{l}0,0589 * * * \\
(0,0013)\end{array}$ \\
\hline Número de nietos & & & & $\begin{array}{c}-0,0035^{* *} \\
(0,0017)\end{array}$ & $\begin{array}{l}0,0059 * * * \\
(0,0017)\end{array}$ & $\begin{array}{c}0,0017 \\
(0,0017)\end{array}$ & \\
\hline Ingreso monetario (M\$ / 2006) & & & & $\begin{array}{l}-0,0001^{* * * *} \\
(0,0000)\end{array}$ & $\begin{array}{l}-0,0001 * * * \\
(0,0000)\end{array}$ & $\begin{array}{l}-0,0001 * * * \\
(0,0000)\end{array}$ & $\begin{array}{l}-0,0001 * * * \\
(0,0000)\end{array}$ \\
\hline Patrimonio activos (MM\$ / 2006 & & & & $\begin{array}{l}0,0003^{* * *} \\
(0,0000)\end{array}$ & $\begin{array}{l}-0,0007 * * * \\
(0,0000)\end{array}$ & $\begin{array}{l}-0,0003 * * * \\
(0,0000)\end{array}$ & \\
\hline Patrimonio deudas (MM\$ / 2006 & & & & $\begin{array}{c}-0,0334 * * * \\
(0,0043)\end{array}$ & $\begin{array}{l}-0,0333 * * * \\
(0,0039)\end{array}$ & $\begin{array}{l}0,0134 * * * \\
(0,0006)\end{array}$ & $\begin{array}{l}0,0076 * * * \\
(0,0004)\end{array}$ \\
\hline Propietario de la vivienda (sí = 1 & & & & $\begin{array}{c}-0,0334 * * * \\
(0,0043)\end{array}$ & $\begin{array}{l}-0,0333 * * * \\
(0,0039)\end{array}$ & & \\
\hline Conocimiento de AFP $(<\%>)$ & & & & & $\begin{array}{l}0,1609 * * * \\
(0,0085)\end{array}$ & $\begin{array}{l}0,1946^{* * * *} \\
(0,0087)\end{array}$ & $\begin{array}{l}0,1173 * * * \\
(0,0079)\end{array}$ \\
\hline $\begin{array}{l}\text { Conocimiento sistema } \\
\text { previsional }(<\%>)\end{array}$ & & & & & $\begin{array}{l}0,0157 * * * \\
(0,0023)\end{array}$ & $\begin{array}{l}0,0168 * * * \\
(0,0024)\end{array}$ & $\begin{array}{l}0,0304 * * * \\
(0,0023)\end{array}$ \\
\hline Conocimiento financiero $(\mathrm{Si}=1)$ & & & & & $\begin{array}{l}0,0672 * * * \\
(0,0051)\end{array}$ & & \\
\hline Zona norte & & & & & $\begin{array}{l}-0,1626^{* * * *} \\
(0,0059)\end{array}$ & & \\
\hline Zona centro & & & & & $\begin{array}{l}-0,2687 * * * \\
(0,0038)\end{array}$ & $\begin{array}{l}-0,1786^{* * * *} \\
(0,0034)\end{array}$ & $\begin{array}{l}-0,2038 * * * \\
(0,0036)\end{array}$ \\
\hline Zona sur & & & & & $\begin{array}{l}-0,15 * * * \\
(0,0047)\end{array}$ & & \\
\hline Años en el mercado laboral & & & & & $\begin{array}{l}-0,0275^{* * *} * \\
(0,0005)\end{array}$ & $\begin{array}{l}-0,0146^{* * *} \\
(0,0002)\end{array}$ & $\begin{array}{l}-0,0262 * * * \\
(0,0006)\end{array}$ \\
\hline Edad de inicio laboral & & & & & $\begin{array}{l}-0,0222 * * * \\
(0,0006)\end{array}$ & & $\begin{array}{l}-0,0234 * * * \\
(0,0006)\end{array}$ \\
\hline Activo laboralmente $($ sí = 1$)$ & & & & & $\begin{array}{c}-0,0020 \\
(0,0028)\end{array}$ & $\begin{array}{l}-0,0201 * * * \\
(0,0029)\end{array}$ & $\begin{array}{l}0,0066^{* *} \\
(0,0027)\end{array}$ \\
\hline Número de observaciones & 134934 & 134934 & 134934 & 134934 & 134934 & 134934 & 134934 \\
\hline Log Likelihood & $-72055,38$ & $-73440,3$ & $-71864,87$ & $-68111,67$ & $-57350,75$ & $-63692,79$ & $-58319,54$ \\
\hline Pseudo - R2 & 0,1668 & 0,1508 & 0,169 & 0,2124 & 0,3368 & 0,2635 & 0,3256 \\
\hline Obs. P & 0,6602 & 0,6602 & 0,6602 & 0,6602 & 0,6602 & 0,6602 & 0,6602 \\
\hline Pred. P & 0,6850 & 0,6814 & 0,6857 & 0,6938 & 0,7438 & 0,7067 & 0,7378 \\
\hline
\end{tabular}

Fuente: elaboración propia sobre la base de la Encuesta de Protección Social (EPS) de 2006.

Nota: $\mathrm{M} \$=$ miles de pesos; $\mathrm{MM}$ \$ = millones de pesos; AIC = Akaike Information Criteria; $\mathrm{BIC}=$ Bayesian Information Criteria. Log Likelihood= Logaritmo de la función de verosimilitud; Obs. $\mathrm{P}=$ Observed probability; Pred. $\mathrm{P}=$ Predicted probability.

Significativo al *10\%; ** Significativo al 5\%; *** Significativo al 1\% (desviación estándar muestral). 
De las estimaciones se obtiene que el efecto de la esperanza de vida en la probabilidad de pensionarse en forma anticipada (parámetro $\Omega_{i}$ ) sea negativo y significativo, con valor estable en todos los modelos (valores entre $-0,38$ y -0,46). Por otra parte, el efecto de la componente combinada entre la esperanza de vida y la aversión al riesgo (parámetro $\Lambda_{i}$ ) resulta ser positivo, aunque su significancia se ve afectada si no se incluye la variable de esperanza de vida. Esto puede deberse a que la aproximación hecha no es satisfactoria, o que la aversión al riesgo también responde a otros factores como el riesgo financiero.

Otras variables como años de escolaridad, género (hombre) y encontrarse activo laboralmente aumentan la utilidad de pensionarse en forma anticipada e influyen positivamente en la probabilidad de adelantar la pensión (véase el cuadro 7). Resultados similares se encuentran en Gustman y Steinmeier (2005) ${ }^{22}$. Sin embargo, el avanzar en edad hace que sea menos probable anticipar la pensión, hecho que es confirmado por las estimaciones de este artículo y evidenciado en otros trabajos (Mitchell y Phillips, 2000).

Si los individuos valoran más su calidad de vida presente que futura, y esto se traduce en pasar más tiempo con sus seres queridos, entonces, el tener hijos y ser casado debería aumentar la probabilidad de pensionarse antes de la edad legal. Los resultados de las estimaciones confirman este hecho, ya que las variables estado civil (en pareja) y tener hijos ${ }^{23}$ resultan ser positivas y significativas, tal se observa en los cuadros 7 y 8 . Esto también se pone en evidencia en Mitchell y Phillips (2000).

Por otra parte, el ingreso, el patrimonio en activos, en deuda y la propiedad de la vivienda, son elementos que podrían estar ligados en el momento de decidir adelantar la pensión, lo que explicaría por qué al quitar una de ellas las otras cambian de signo, mas no de significancia; por ende, deben ser consideradas (véanse los cuadros 7 y 8 ).

\section{b) Principales hallazgos}

Las personas, al ir avanzando en edad, pueden valorar de distinta manera los años que les quedan por vivir, lo que estará condicionado tanto por las

\footnotetext{
${ }^{22}$ Sus estimaciones se basan en explicar la influencia de la seguridad social y otros factores en la decisión de pensión y no en la de pensionarse de forma anticipada; sin embargo, sus alcances dan apoyo a las estimaciones hechas en este artículo.

${ }^{23}$ El considerar hijos, sin hacer distinción de su procedencia, hace más significativa la variable que solo considerar hijos dentro del hogar.
}

preferencias individuales como por la calidad de vida futura que se espere de los años venideros. La incertidumbre vinculada a estos años de vida futura hace que la aversión al riesgo tenga un papel en las decisiones de consumo. En particular, lo que se ha propuesto en este artículo es que los individuos tendrían distintas tasas de descuento intertemporal, dependiendo de cómo valoran su esperanza de vida futura y de la aversión al riesgo, lo que afectaría a la decisión de pensión anticipada. Los resultados encontrados ponen en evidencia que aquellas personas que descuentan más el futuro debido a una menor valoración de la esperanza de vida futura, a una mayor aversión al riesgo o a ambas, tienen más probabilidades de pensionarse de manera anticipada.

El disfrutar tiempo de calidad en el presente, sobre todo al aproximarse la edad de jubilación, no es completamente desconocido por el mercado de las pensiones, ya que en el momento en que un individuo manifiesta inquietud por pensionarse, los asesores de las diferentes instituciones existentes en este mercado lo inducen a reflexionar sobre el hecho de compartir más con su familia en el presente, y de esta manera lo hacen más consciente de su mayor descuento del futuro. Por lo tanto, ambientes económicos desfavorables, deterioro progresivo de la salud, una familia con quien compartir, y poco o nada de conocimientos acerca del mercado de las pensiones podrían facilitar una percepción pesimista del futuro, dando más valor al uso del tiempo en el presente y logrando una mayor tasa de descuento, o al menos lo suficiente como para que la decisión óptima sea pensionarse antes de la edad legal.

Si bien Guiso y Paiella (2006) encuentran empíricamente que la aversión al riesgo contribuye a explicar una serie de decisiones individuales como emprendimiento, portafolio, demanda de seguros, inversión en educación, migración, cambios de trabajo y estado de salud, no hay antecedentes de su efecto en la decisión de pensión anticipada. En este contexto, los resultados de este trabajo muestran que la aversión al riesgo por sí sola no es un elemento determinante de la decisión de pensión anticipada, pero sí lo es al combinarse con percepciones futuras de las personas (véase el cuadro 7 , modelos ii y iii). Además, la repercusión marginal de la aversión al riesgo en la probabilidad de pensión anticipada es sustancialmente mayor que la de la edad, la escolaridad, la presencia de la pareja en el hogar o el número de hijos (véase el cuadro 7 , modelos vi y vii). 


\section{$\mathrm{V}$}

\section{Conclusiones}

En este artículo se ha presentado evidencia de que aquellos que se pensionan de forma anticipada poseen una mayor tasa de descuento intertemporal, lo que se explicaría por la mayor valoración de los años que quedan por vivir en el presente y por la aversión al riesgo que condiciona la incertidumbre de esos años de vida adicional. Si bien en la literatura la aversión al riesgo ha sido vinculada a una serie de decisiones individuales, este artículo es el primero en que lo hace respecto de la decisión de pensión anticipada.

Al considerar la edad promedio de los entrevistados y su esperanza de vida, tanto el grupo de los pensionados a la edad legal, como el de los que lo hicieron de forma anticipada, creen que su esperanza de vida media es de aproximadamente 80 años.

Las estimaciones revelaron que ser el jefe de hogar $\mathrm{y}$ tener un mayor número de hijos influye positiva y significativamente en la probabilidad de adelantar la pensión. Esto podría deberse a las urgentes necesidades de contribuir con recursos al hogar en el presente, que hacen que la valoración actual de dichos recursos sea mayor que la futura, lo que resulta en desmedro de una mejor pensión futura.

Se encontró evidencia robusta de que la valoración de la esperanza de vida futura es diferente entre los grupos de pensionados, lo que se traduce en un menor factor de descuento intertemporal en aquellos que tienen una mayor valoración de su esperanza de vida en el presente que en el futuro, y en consecuencia adelantan su pensión.

Además, se encontró evidencia de que mientras más alto es el nivel de aversión al riesgo, más se exacerba la impaciencia por pensionarse de forma anticipada, lo que podría deberse a la incertidumbre de poder disfrutar de una mejor calidad de vida en el futuro. Existiría entonces un mayor beneficio al descontar más la utilidad futura, prefiriéndose disfrutar de una mejor calidad de vida presente. Estos efectos son importantes en tamaño en relación con otras variables como edad, escolaridad y estructura del hogar.

De esta manera, en este artículo se aporta evidencia en favor de la hipótesis de heterogeneidad en tasas de descuento intertemporal vinculada a las expectativas de vida futura, lo que se acentúa ante la incertidumbre relacionada con la calidad de vida futura. Como extensión de estas conclusiones, se podría predecir que situaciones tales como crisis económicas, mayor incertidumbre de poder disfrutar tiempo de calidad en el futuro y pequeñas perturbaciones (shocks) monetarias, pueden aumentar la probabilidad de pensionarse anticipadamente. 
$\operatorname{ANEXOA}$

\section{Cuadros complementarios}

La Encuesta de Protección Social (EPS) del año 2006 presenta en el módulo J tres preguntas (de la j1_1 a la j1_3), las que agrupan a los entrevistados en cuatro niveles de aversión al riesgo, que van de un bajo nivel de aversión (1) a un alto nivel de aversión (4).

CUADRO A.1

Distribución poblacional, por sexo, activo y pensionado, según nivel de aversión al riesgo

\begin{tabular}{|c|c|c|c|c|c|c|c|}
\hline \multirow{2}{*}{ Nivel de aversión } & \multirow{2}{*}{$\begin{array}{c}\text { Distribución } \\
\text { población }\end{array}$} & \multicolumn{2}{|c|}{ Sexo } & \multicolumn{2}{|c|}{ Trabajando } & \multicolumn{2}{|c|}{ Pensionados } \\
\hline & & Mujeres & Hombres & No & Sí & No & Sí \\
\hline 2 (Medio bajo) & 985508 & 464222 & 521286 & 398050 & 587458 & 905571 & 79937 \\
\hline 3 (Medio alto) & 750826 & 361057 & 389769 & 327635 & 423191 & 708212 & 42614 \\
\hline 4 (Alto) & 7478283 & 3975611 & 3502672 & 3360807 & 4117476 & 6789315 & 688968 \\
\hline Total & 11492732 & 5765784 & 5726948 & 4958321 & 6534411 & 10549199 & 943533 \\
\hline
\end{tabular}

Fuente: elaboración propia sobre la base de la Encuesta de Protección Social (EPS) de 2006.

CUADRO A. 2

Participación de los afiliados dentro de los sistemas de pensiones

\begin{tabular}{|c|c|c|c|c|}
\hline \multicolumn{5}{|c|}{ Sistema previsional } \\
\hline \multirow{2}{*}{ Sistema } & \multicolumn{2}{|c|}{ Población } & \multicolumn{2}{|c|}{ Pensionados } \\
\hline & $\mathrm{N}^{\mathrm{o}}$ & Porcentaje & $\mathrm{N}^{\mathrm{o}}$ & Porcentaje \\
\hline AFP & 7550278 & 88,5 & 338274 & 41,3 \\
\hline INP & 849219 & 10,0 & 421799 & 51,5 \\
\hline CAPREDENA & 25260 & 0,3 & 9123 & 1,1 \\
\hline DIPRECA & 26228 & 0,3 & 5173 & 0,6 \\
\hline Otro sistema & 81157 & 1,0 & 44543 & 5,4 \\
\hline Total & 8532142 & 100,0 & 818912 & 100,0 \\
\hline
\end{tabular}

Fuente: elaboración propia sobre la base de la Encuesta de Protección Social (EPS) de 2006. Población que autorreporta el sistema previsional al que pertenece.

AFP: Administradoras de Fondos de Pensiones.

INP: Instituto de Normalización Previsional.

CAPREDEna: Caja de Previsión de la Defensa Nacional.

DIPRECA: Dirección de Previsión de Carabineros. 


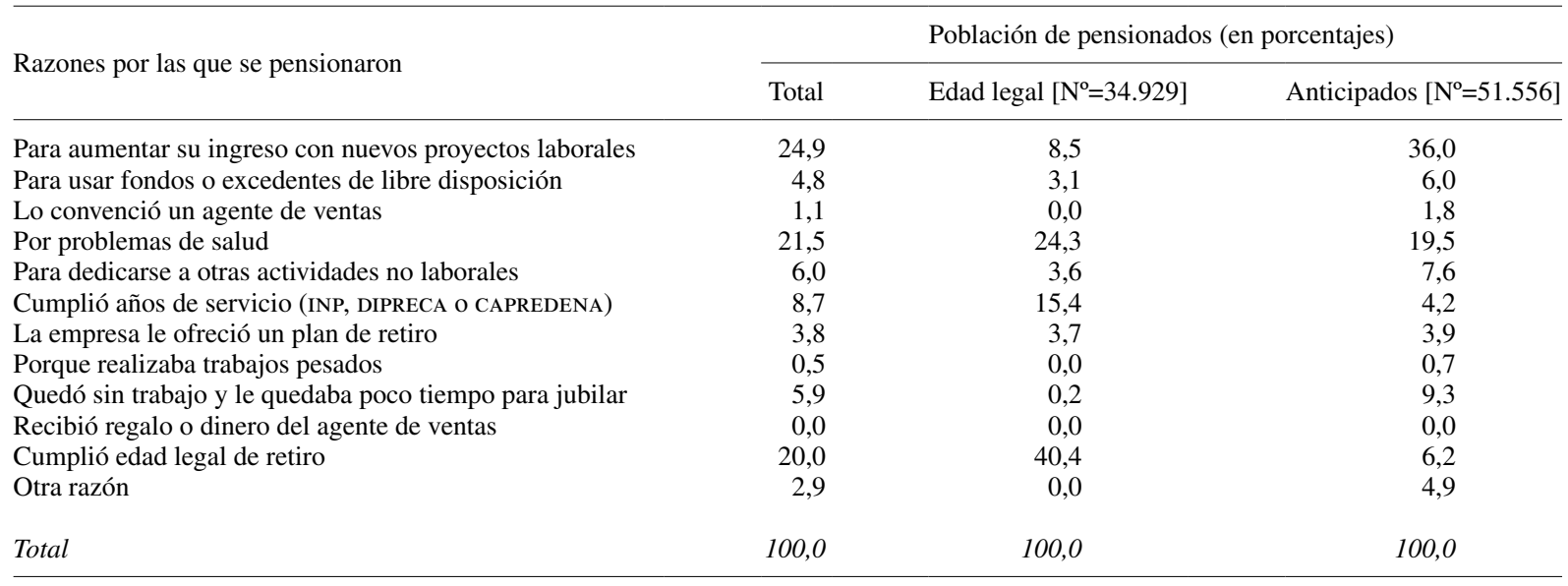

Fuente: elaboración propia sobre la base de la Encuesta de Protección Social (EPS) de 2006.

INP: Instituto de Normalización Previsional.

CAPREDENA: Caja de Previsión de la Defensa Nacional.

DIPRECA: Dirección de Previsión de Carabineros.

Au, A., O. Mitchell y J. Phillips (2005), "Saving shortfalls and delayed retirement", Working Paper, N WP-094, Michigan, Michigan Retirement Research Center, Universidad de Michigan.

Bleichrodt, H. y J. Quiggin (1999), "Life-cycle preferences over consumption and health: when is cost-effectiveness analysis equivalent to cost-benefit analysis?", Journal of Health Economics, vol. $18, \mathrm{~N}^{\circ} 6$, Amsterdam, Elsevier.

Chai, J. y otros (2009), "Extending life cycle models of optimal portfolio choice: integrating flexible work, endogenous retirement, and investment decisions with lifetime payouts", NBER Working Paper, $\mathrm{N}^{\mathrm{o}} 15079$, Cambridge, Massachusetts, National Bureau of Economic Research.

Deaton, A. y J. Muellbauer (1980), "An almost ideal demand system", American Economic Review, vol. 70, №3, Nashville, Tennessee, American Economic Association.

Diamond, P. y B. Köszegi (2003), "Quasi-hyperbolic discounting and retirement", Journal of Public Economics, vol. 87, № 9-10, Amsterdam, Elsevier.

Engen, E.M, W.G. Gale y C. Uccello (1999), "The adequacy of retirement saving", Brookings Papers en Economic Activity, vol. 30, $\mathrm{N}^{\circ}$ 2, Washington, D.C., The Brookings Institution.

French, E. (2005), "The effects of health, wealth, and wages on labour supply and retirement behavior", Review of Economic Studies, vol. $72, \mathrm{~N}^{\circ} 2$, John Wiley \& Sons.

Guiso, L. y M. Paiella (2008), "Risk aversion, wealth, and background risk", Journal of the European Economic Association, vol. 6, $\mathrm{N}^{\mathrm{o}}$ 6, Cambridge, Massachusetts, MIT Press.

(2006), "The role of risk aversion in predicting individual behavior", Insurance: Theoretical Analysis and Policy Implications, P. Chiappori y C. Gollier (eds.), CESifo.
Gustman, A. y T. Steinmeier (2005), "The social security early entitlement age in a structural model of retirement and wealth", Journal of Public Economics, vol. 89, No 2-3, Amsterdam, Elsevier.

Hairault, J-O., F. Langot y T. Sopraseuth (2010), "Distance to retirement and older workers' employment: the case for delaying the retirement age", Journal of the European Economic Association, vol. 8, $\mathrm{N}^{\circ}$ 5, Cambridge, Massachusetts, MIT Press, septiembre.

Hammitt, J., K. Haninger y N. Treich (2005), "The effects of health and longevity on risk tolerance", Boston, Harvard Center for Risk Analysis.

Hensher, D., P. Barnard y P. Truong (1988), "The role of stated preference methods in studies of travel choice", Journal of Transport Economics and Policy, vol. 22, $\mathrm{N}^{\circ}$ 1, Bath, Universidad de Bath.

Mitchell, O. y J. Phillips (2000), "Retirement responses to early social security benefit reductions", Working Paper, $\mathrm{N}^{\circ}$ WP-006, Michigan, Michigan Retirement Research Center, Universidad de Michigan.

Nalebuff, B. y R. Zeckhauser (1985), "Pensions and the retirement decision", Pensions, Labor, and Individual Choice, David A. Wise (comp.), Cambridge, Massachusetts, National Bureau of Economic Research.

Nordhaus, W.D. (2002), "The health of nations: the contribution of improved health to living standards", NBER Working Paper, $\mathrm{N}^{\mathrm{o}}$ 8818, Cambridge, Massachusetts, National Bureau of Economic Research.

Poterba, J., J. Rauh y S. Venti (2005), "Utility evaluation of risk in retirement saving accounts", Analyses in the Economics of Aging, Chicago, University of Chicago Press. 